\title{
Two-photon Photoselection: An Irreducible Tensor Analysis
}

\author{
David L. Andrews and Bridget S. Webb† \\ School of Chemical Sciences, University of East Anglia, Norwich NR4 7TJ, UK
}

\begin{abstract}
A detailed symmetry analysis of two-photon photoselection is presented. The adoption of irreducible tensor methods facilitates the characterization of two-photon excited states probed either by fluorescence or by the absorption of a third photon from a probe laser beam. The theory also enables information on any intervening rotational motion to be derived from suitable polarization studies. A new generalized scheme for the symmetry classification of multiphoton transitions is applied to the photoselection process.
\end{abstract}

\section{Introduction}

Photoselection is a method of investigating a molecular system using a polarized beam of light to create a preferentially orientated population of excited molecules. Significant work in this area was first carried out in 1934 by Perrin, in order to investigate the hydrodynamics of molecular rotation. The term photoselection seems to have originated with Albrecht, ${ }^{1}$ who in 1961 first gave a detailed outline of the theory, and suggested that photoselection techniques should be more widely used. In the same year Feofilov ${ }^{2}$ gave a description of photoselection as a probe of specific molecular electronic properties, but it was not until mode-locked lasers were widely available that photoselection became a more general technique. The difficulties of the spectroscopic examination of very short-lived excited states were overcome by the use of picosecond laser pulses, thus extending the field of study from long-lived metastable electronic states and stable photoproducts in rigid matrices to almost any state with a picosecond or longer lifetime. Photoselection has since become an important tool in the study of many chemical and biological systems. ${ }^{3-9}$

Some early multiphoton photoselection studies were carried by Dowley et al. ${ }^{10}$ These experiments involved measurement of the polarization of fluorescence following two-photon excitation with linearly polarized light. McClain ${ }^{11,12}$ subsequently developed much of the basic theory for two-photon photoselection by expressing the three-photon molecular response tensor as the product of a second-rank absorption tensor and a fluorescence transition moment. The next milestone in the theory was the publication in 1978 of a paper by Magde, ${ }^{13}$ in which the theory of photoselection was re-derived and extended to the non-linear regime, including the saturation effects which are prominent when intense laser beams are used.

Since Magde's paper, Albrecht and co-workers ${ }^{14,15}$ have studied the $B_{2 u} \leftarrow A_{1 g}$ transition in benzene using multiphoton photoselection with both two- and three-photon excitation. Cable and Albrecht ${ }^{16}$ have also written on the theory of threephoton photoselection, demonstrating that five unique transition tensor invariants characterize the symmetry of the threephoton absorption stage. Melikechi and Allen ${ }^{17}$ have investigated the angular distribution of fluorescence emitted following two-photon absorption, showing that in some two-photon absorption experiments more information can be gained by observing the fluorescence at angles other than $\pi / 2$ to the excitation beams, the angle most commonly used. Kummel et al. ${ }^{18,19}$ have recently demonstrated the use of multiphoton photoselection techniques in the determination of the population and orientation of prepared states using two-photon excitation with elliptically polarized light. Docker ${ }^{20}$ has also described in general terms how the alignment of molecular angular momenta can be measured by multiphoton methods.

The excited state produced in photoselection studies may be examined by any spectroscopic method, although for experiments using mode-locked pulses the photoselected distribution is usually studied using time-resolved absorption and emission techniques before relaxation can occur. ${ }^{21}$ The process of rotational relaxation of dyes and other polyatomic molecules mostly occurs on a picosecond timescale and so is usually investigated by absorption spectroscopy, but in some cases fluorescent decay may also be used if it is sufficiently fast. This is the case with cyanine dyes, phthalocyanines, metalloporphyrins with transitionmetal centres and also triphenylmethane dyes. Whilst the measurement of transient absorption is usually less sensitive than a technique with a zero background, such as fluorescence, polarization-selective photoselection experiments can have a zero background, ${ }^{5}$ and such experiments can be used to measure rotational correlation times directly.

In general, the excited state populated by a multiphoton photoselection process can be probed either by its fluorescence or by absorption of one further photon from a probe laser beam. Both cases are treated in this paper within a common formalism based on the methods of molecular quantum electrodynamics. By employing irreducible tensor methods it is shown how polarization studies can provide important information on the extent of molecular rotation between the two transitions. Measurements based on the use of pulsed lasers with pump/probe instrumentation should thus furnish a greater understanding of excited-state dynamics.

\section{Dynamics of Multiphoton Photoselection}

\subsection{Perturbation Theory}

To calculate theoretical rates of absorption, and hence polarization ratios for multiphoton photoselection, the average transition rate for a general $n$-photon event is needed. This is first derived from the transition rate for a single molecule localized in a specific orientation, and then averaged over all possible orientations. This is based on the normal assumption that the quantum structure of rotational motion is not resolved in the liquid phase; rich rotational structure is of course obtainable in gas-phase multiphoton studies, as typified by the beautiful results of Dixon and co-workers [see for example ref. (22)]. 
The quantum electrodynamical Hamiltonian for a system comprising an ensemble of molecules in the presence of radiation may be expressed as

$$
H=H_{\mathrm{mol}}+H_{\mathrm{rad}}+H_{\mathrm{int}}
$$

where

$$
\begin{aligned}
& H_{\mathrm{mol}}=\sum_{\xi}\left(\frac{1}{2 m} \sum_{\alpha} p_{\alpha}^{2}(\xi)+V(\xi)\right) \\
& H_{\mathrm{rad}}=\frac{1}{2} \int\left(\frac{d^{\perp 2}}{\varepsilon_{0}}+c^{2} \varepsilon_{0} b^{2}\right) \mathrm{d}^{3} r \\
& H_{\mathrm{int}}=-\frac{1}{\varepsilon_{0}} \sum_{\xi} \mu(\xi) \cdot d^{\perp}\left(R_{\xi}\right)-\cdots .
\end{aligned}
$$

The first term, $H_{\text {mol }}$, represents the sum over each molecule $\xi$ at position $\boldsymbol{R}_{\xi}$ of its normal non-relativistic Schrödinger operator, $p_{\alpha}$ denoting the momentum of the electron $\alpha$, and $V(\xi)$ the total intramolecular Coulombic potential energy. The subsequent term, $H_{\mathrm{rad}}$, represents the radiation Hamiltonian expressed in terms of the transverse displacement field operator $d^{\perp}(r)$ and the magnetic field operator $b(r)$. The interaction Hamiltonian $H_{\text {int }}$ in general consists of a series of terms. For the calculations leading to the results given in this paper, the electric-dipole approximation is employed for each molecule. The justification is the fact that provided each molecule-photon interaction is electric dipole-allowed, the contributions from other terms in $H_{\text {int }}$ are considerably smaller in magnitude, the electric quadrupole and magnetic dipole being smaller by a factor of approximately $\alpha$ (the fine-structure constant). Finally, the mode expansion of the electric displacement field operator in a quantization volume $V$ is as follows:

$$
d^{\perp}(\boldsymbol{r})=\mathrm{i} \sum_{\boldsymbol{k}, \lambda}\left(h c k \varepsilon_{0} / 2 V\right)^{1 / 2} \times\left[\boldsymbol{e}^{(\lambda)}(\boldsymbol{k}) a^{(\lambda)}(\boldsymbol{k}) \exp (\mathrm{i} \boldsymbol{k} \cdot \boldsymbol{r})-\bar{e}^{(\lambda)}(\boldsymbol{k}) a^{\dagger(\lambda)}(\boldsymbol{k}) \exp (-\mathrm{i} \boldsymbol{k} \cdot \boldsymbol{r})\right]
$$

where $a^{(\lambda)}(k)$ and $a^{\dagger(\lambda)}(k)$ are the annihilation and creation operators for the radiation mode with wavevector $k$ and polarization $\lambda$. Since these operators appear linearly in $d^{\perp}(r)$, each electric-dipole interaction can be associated with either the absorption or emission of a single photon.

We start with the familiar generalized expression for the time-dependent probability $P_{\mathrm{f}}(t)$, associated with a single-photon transition $|f\rangle \leftarrow|i\rangle$ of energy $E_{f i}=\hbar \omega_{f i}$, induced by radiation of circular frequency $\omega$ :

$$
P_{f}(t)=\frac{\left|M_{f i}\right|^{2}}{\hbar^{2}} \frac{\sin ^{2} \frac{1}{2}\left(\omega-\omega_{f i}\right) t}{\left(\omega-\omega_{f i}\right)^{2}}
$$

where $M_{f i}$ is the transition matrix element with its usual perturbation series expansion:

$$
M_{f i}=\left\langle f\left|H_{\mathrm{int}}\right| i\right\rangle+\sum_{r} \frac{\left\langle f\left|H_{\mathrm{int}}\right| r\right\rangle\left\langle r\left|H_{\mathrm{int}}\right| i\right\rangle}{E_{i}-E_{r}}+\sum_{r, s} \frac{\left\langle f\left|H_{\mathrm{int}}\right| s\right\rangle\left\langle s\left|H_{\mathrm{int}}\right| r\right\rangle\left\langle r\left|H_{\mathrm{int}}\right| i\right\rangle}{\left(E_{i}-E_{s}\right)\left(E_{i}-E_{r}\right)}+\cdots
$$

with $H_{\text {int }}$ the perturbation operator. The probability given by eqn (6) peaks sharply at $\omega=\omega_{f i}$, corresponding to the absorption of a photon, although there is a non-zero probability at $\omega \neq \omega_{f i}$, associated with the time-energy uncertainty principle. The oscillatory nature of the transition probability is not normally seen, but has been observed in molecular-beam experiments by Dyke et al. ${ }^{23}$ Taking the limit of $P_{f}(t)$ as $\omega_{f i} \rightarrow \omega$ gives:

$$
P_{f}(t)=\frac{\left|M_{f i}\right|^{2} t^{2}}{\hbar^{2}}
$$

This result applies to a system with discrete molecular energy levels and with radiation of a finite linewidth. When there is a finite lifetime for the excited state $|f\rangle$, it is usual to average over a density of state $\rho_{f}$, with the result

$$
P_{f}(t)=\frac{2 \pi}{\hbar}\left|M_{f i}\right|^{2} \rho_{f} t
$$

Again $M_{f i}$ is the molecular transition matrix element and $\rho_{f}$ is the density of states. This result is the Fermi 'Golden' rule. The two results (8) and (9) are appropriate for different stages of a multiphoton photoselection process.

\subsection{Rate Equations for Photoselection}

In the photoprocesses to be examined below, photons from the pump beams excite the system from the ground state $|i\rangle$ to a discrete excited state $|f\rangle$, then either another photon is absorbed from a probe beam or one is emitted, taking the system to a final state $|g\rangle$ (fig. 1). The exact resonance equation gives the probability that any initial $m$-photon transition $|f\rangle \leftarrow|i\rangle$ will occur,

$$
P_{1}^{m \phi}=\frac{\left|M_{f i}^{m \phi}\right|^{2} t^{2}}{\hbar^{2}}
$$

with

$$
M_{f i}^{m \phi}=-\mathrm{i}^{m}\left(n_{1} n_{2} \cdots n_{m} \omega_{1} \omega_{2} \cdots \omega_{m}\right)^{1 / 2} \times\left(\frac{\hbar}{2 \varepsilon_{0} V}\right)^{m / 2} S_{i_{1}, i_{2} \cdots i_{m}} e_{1_{i, 1}} e_{2_{i, 2}} \cdots e_{m_{i, m}} .
$$




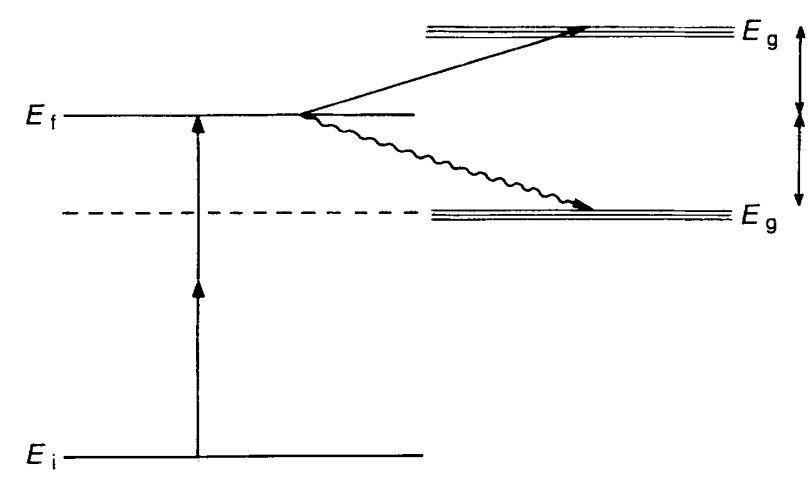

Fig. 1. Schematic representation of two-photon photoselection. Two photons are absorbed in the transition $|i\rangle \rightarrow|f\rangle$; the molecule then proceeds to a final state $|g\rangle$ either through fluoresence or through absorption of a third photon from a probe laser beam.

Since the secondary stage of the process terminates in a state $|g\rangle$ with which decay channels are normally associated (and indeed are often involved in detection of the process), the Fermi rule applies,

$$
P_{2}^{1 \phi}=\frac{2 \pi}{\hbar}\left|M_{g f}^{1 \phi}\right|^{2} \rho_{g} t
$$

with

$$
M_{g f}^{1 \phi}=-\mathrm{i}\left(\frac{n_{m+1} \omega_{m+1} h}{2 \varepsilon_{0} V}\right)^{1 / 2} \mu_{i_{m+1}}^{g f} e_{m+1} 1_{i, m+1}
$$

The two probabilities given by eqn (10) and (12) now need to be combined in order to obtain an overall probability or rate of transition. For simplicity, the following derivation is based on the assumption of CW irradiation; the procedure for incorporating the time-dependent effects of pulsed radiation is described in detail elsewhere. ${ }^{24}$ The temporal characteristics of laser pulsing are in any case associated with timescales normally well in excess of the optical cycle lengths.

If $P_{1}(\tau)$ represents the fractional number of molecules that have undergone the initial transition $|f\rangle \leftarrow|i\rangle$ after a time $\tau$ has elapsed, the rate of this transition, $R_{1}$, is:

$$
R_{1}=\frac{\mathrm{d}}{\mathrm{d} \tau} P_{1}(\tau)
$$

Using eqn (11) and putting

$$
A_{1}=\frac{\left|M_{f i}^{n \phi}\right|^{2}}{\hbar^{2}}
$$

then

$$
\begin{aligned}
& R_{1}(\tau)=2 A_{1} \tau \\
& P_{1}(\tau)=A_{1} \tau^{2}
\end{aligned}
$$

and using eqn (12) with

$$
A_{2}=\frac{2 \pi}{\hbar}\left|M_{g f}^{1 \phi}\right|^{2} \rho_{g}
$$

we obtain

$$
\begin{gathered}
R_{2}=A_{2} \\
P_{2}(\tau)=A_{2} \tau .
\end{gathered}
$$

Assuming that the number of molecules in the ground state $|i\rangle$ does not change significantly from $N$ throughout the process, then the rate equations for the system are:

$$
\begin{aligned}
\frac{\mathrm{d} N_{i}(t)}{\mathrm{d} t} & =-N R_{1}(t) \\
\frac{\mathrm{d} N_{f}(t)}{\mathrm{d} t} & =N R_{1}(t)-N_{f}(t) R_{2} \\
\frac{\mathrm{d} N_{g}(t)}{\mathrm{d} t} & =N_{f}(t) R_{2}
\end{aligned}
$$


Using the complementary function $\beta \exp \left(-R_{2} t\right)$ and the particular integral $\gamma t+\delta$ to solve the second of these differential equations subject to the initial condition $N_{f}(0)=0$, we obtain the result:

$$
N_{f}(t)=\frac{2 A_{1} N}{A_{2}}\left(\frac{\exp \left(-A_{2} t\right)-1}{A_{2}}+t\right)
$$

Using this in the third rate expression of eqn (21) we obtain

$$
\begin{aligned}
N_{g}(t) & =\int_{0}^{\tau} A_{2} \frac{2 A_{1} N}{A_{2}}\left(\frac{\exp \left(-A_{2} t\right)-1}{A_{2}}+t\right) \mathrm{d} t \\
& =A_{2} \frac{2 A_{1} N}{A_{2}} \int_{0}^{\tau} \frac{1}{A_{2}}\left[\exp \left(-A_{2} t\right)-1\right]+t \mathrm{~d} t \\
& =2 N A_{1}\left[-\frac{1}{A_{2}}\left(\frac{\exp \left(-A_{2} t\right)}{A_{2}}-t\right)+\frac{t^{2}}{2}\right]_{0}^{\tau} \\
& =2 N A_{1}\left[-\frac{1}{A_{2}}\left(\frac{\exp \left(-A_{2} \tau\right)-1}{A_{2}}-\tau\right)+\frac{\tau^{2}}{2}\right] .
\end{aligned}
$$

However,

$$
\begin{aligned}
P(\tau) & =\frac{N_{g}(\tau)}{N} \\
& =2 A_{1}\left[-\frac{1}{A_{2}}\left(\frac{\exp \left(-A_{2} \tau\right)-1}{A_{2}}-\tau\right)+\frac{\tau^{2}}{2}\right]
\end{aligned}
$$

Expanding the exponential in this yields:

$$
\begin{aligned}
P(\tau) & =2 A_{1}\left\{-\frac{1}{A_{2}}\left[\frac{1}{A_{2}}\left(1-A_{2} \tau+\frac{A_{2}^{2} \tau^{2}}{2}-\frac{A_{2}^{3} \tau^{3}}{6}+\cdots-1\right)+\tau\right]+\frac{\tau^{2}}{2}\right\} \\
& \approx \frac{A_{1} A_{2} \tau^{3}}{3}
\end{aligned}
$$

Any further powers of $A_{2} \tau$ in the expansion will be very small since $A_{2} t \ll 1$. It is worth noting that $\tau^{3}$ characteristics have also been noted in connection with resonance atomic photoionization processes. ${ }^{25-27}$ Going back to eqn (17) and (20), the overall rate therefore becomes:

$$
P(\tau)=\int_{0}^{\tau} P_{1}(t) R_{2} \mathrm{~d} t
$$

and in general the integration will be performed over a time corresponding to the pulse length of the laser.

\subsection{Two-photon Photoselection}

The kinetic characteristics of two-photon photoselection generally lie between two extremes: (1) absorption of two photons and then interaction with another photon after rotational relaxation has had time to occur, and (2) a concerted three-photon process. The transition probabilities for these two cases will first be determined and then an intermediate case considered.

\subsubsection{Sequential Process}

Two pump photons are absorbed in general from two different laser beams, and another is either emitted or absorbed subsequently. For the pump $|f\rangle \leftarrow|i\rangle$ transition

$$
\begin{aligned}
P_{i}^{2 \phi} & =\frac{\left\langle\left|M_{f i}^{2 \phi}\right|^{2}\right\rangle t^{2}}{\hbar^{2}} \\
\left\langle\left|M_{f i}^{2 \phi}\right|^{2}\right\rangle & =\left\langle\left|\left(n_{1} n_{2} \omega_{1} \omega_{2}\right)^{1 / 2}\left(\frac{\hbar}{2 \varepsilon_{0} V}\right) S_{i j} e_{1_{i}} e_{2_{j}}\right|^{2}\right\rangle \\
& =\left\langle\left|\left(\frac{n_{1} \omega_{1} \hbar}{V}\right)^{1 / 2}\left(\frac{n_{2} \omega_{2} \hbar}{V}\right)^{1 / 2} \frac{1}{2 \varepsilon_{0}} S_{i j} e_{1_{i}} e_{2_{j}}\right|^{2}\right\rangle
\end{aligned}
$$


where $S_{i j}$ is the normal two-photon absorption tensor. Since the irradiance of each pump beam is given by $I=n \hbar c \omega / V$, we then have:

$$
\left\langle\left|M_{f i}^{2 \phi}\right|^{2}\right\rangle=\frac{I_{1} I_{2}}{4 \varepsilon_{0}^{2} c^{2}}\left\langle\left|S_{i j} e_{1 i} e_{2 j}\right|^{2}\right\rangle
$$

In the special case where both pump photons derive from a single laser beam, the quantity $I_{1} I_{2}$ is replaced by $g^{(2)} I^{2}$, where $I$ is the irradiance and $g^{(2)}$ the degree of second-order coherence. To proceed further we now need to evaluate the rotational average of eqn (28) as expressed by the angular brackets. Using the terminology and results of ref. (28) we then obtain;

$$
\begin{aligned}
\left\langle\left|M_{f i}^{2 \phi}\right|^{2}\right\rangle & =\frac{I_{1} I_{2}}{4 \varepsilon_{0}^{2} c^{2}}\left\langle l_{i \lambda} l_{j \mu} l_{k v} l_{l o} S_{\lambda \mu} \bar{S}_{v o} e_{1_{i}} \bar{e}_{1_{k}} e_{2_{j}} \bar{e}_{2_{l}}\right\rangle \\
& =\frac{I_{1} I_{2}}{4 \varepsilon_{0}^{2} c^{2}} I_{i j k l: \lambda \mu v o}^{(4)} S_{\lambda \mu} \bar{S}_{v o} e_{1_{i}} \bar{e}_{1_{k}} e_{2_{j}} \bar{e}_{2_{l}} \\
& =\frac{I_{1} I_{2}}{120 \varepsilon_{0}^{2} c^{2}}\left(\begin{array}{l}
\left(e_{1} \cdot e_{2}\right)\left(\bar{e}_{1} \cdot \bar{e}_{2}\right) \\
\left(e_{1} \cdot \bar{e}_{1}\right)\left(e_{2} \cdot \bar{e}_{2}\right) \\
\left(e_{1} \cdot \bar{e}_{2}\right)\left(e_{2} \cdot \bar{e}_{1}\right)
\end{array}\right)\left(\begin{array}{rrr}
4 & -1 & -1 \\
-1 & 4 & -1 \\
-1 & -1 & 4
\end{array}\right)\left(\begin{array}{l}
S_{\lambda \lambda} \bar{S}_{\mu \mu} \\
S_{\lambda \mu} \bar{S}_{\lambda \mu} \\
S_{\lambda \mu} \bar{S}_{\mu \lambda}
\end{array}\right)
\end{aligned}
$$

where $e_{1}$ and $e_{2}$ denote the polarization vectors of the two beams.

In order to study the selection rules for this process it is most convenient to recast the above result in terms of irreducible Cartesian tensors. To do so requires use of the following relations:

$$
\begin{aligned}
S_{\lambda \lambda} \bar{S}_{\mu \mu} & =3 S_{\lambda \mu}^{(0)} \bar{S}_{\lambda \mu}^{(0)} \\
S_{\lambda \mu} \bar{S}_{\lambda \mu} & =S_{\lambda \mu}^{(0)} \bar{S}_{\lambda \mu}^{(0)}+S_{\lambda \mu}^{(1)} \bar{S}_{\lambda \mu}^{(1)}+S_{\lambda \mu}^{(2)} \bar{S}_{\lambda \mu}^{(2)} \\
S_{\lambda \mu} \bar{S}_{\mu \lambda} & =S_{\lambda \mu}^{(0)} \bar{S}_{\mu \lambda}^{(0)}+S_{\lambda \mu}^{(1)} \bar{S}_{\mu \lambda}^{(1)}+S_{\lambda \mu}^{(2)} \bar{S}_{\mu \lambda}^{(2)} \\
& =S_{\lambda \mu}^{(0)} \bar{S}_{\lambda \mu}^{(0)}-S_{\lambda \mu}^{(1)} \bar{S}_{\lambda \mu}^{(1)}+S_{\lambda \mu}^{(2)} \bar{S}_{\lambda \mu}^{(2)}
\end{aligned}
$$

where $S^{(0)}$ is a weight-0 tensor transforming like a scalar, $S^{(1)}$ a weight-1 tensor transforming like a pseudo-vector (even parity) and $S^{(2)}$ has the transformation properties of a symmetric traceless rank-two tensor. Hence from eqn (26) and (29)-(32) we obtain:

$$
P_{2}^{2 \phi}=\frac{I_{1} I_{2}}{120 \varepsilon_{0}^{2} c^{2} \hbar^{2}}\left(\begin{array}{c}
\left(e_{1} \cdot e_{2}\right)\left(\bar{e}_{1} \cdot \bar{e}_{2}\right) \\
1 \\
\left(e_{1} \cdot \bar{e}_{2}\right)\left(e_{2} \cdot \bar{e}_{1}\right)
\end{array}\right)\left(\begin{array}{rrr}
10 & 0 & -2 \\
0 & 5 & 3 \\
0 & -5 & 3
\end{array}\right)\left(\begin{array}{l}
S_{\lambda \mu}^{(0)} \bar{S}_{\lambda \mu}^{(0)} \\
S_{\lambda \mu}^{(1)} \bar{S}_{\lambda \mu}^{(1)} \\
S_{\lambda \mu}^{(2)} \bar{S}_{\lambda \mu}^{(2)}
\end{array}\right) t^{2}
$$

For the secondary transition $|g\rangle \leftarrow|f\rangle$ we have

$$
\begin{aligned}
P_{2}^{1 \phi} & =\frac{2 \pi}{\hbar}\left\langle\left|M_{g f}^{1 \phi}\right|^{2}\right\rangle \rho_{g} t \\
& =\frac{2 \pi}{\hbar}\left\langle\left|-\mathrm{i}\left(\frac{n_{3} \omega_{3} \hbar}{2 \varepsilon_{0} V}\right)^{1 / 2} \mu_{m}^{g f} e_{3 m}\right|^{2}\right\rangle \rho_{g} t \\
& =\frac{2 \pi I_{3}}{2 \varepsilon_{0} \hbar c}\left\langle\mu_{m}^{g f} \bar{\mu}_{n}^{g f} e_{3 m} \bar{e}_{3 n}\right\rangle \rho_{g} t \\
& =\frac{\pi I_{3}}{\varepsilon_{0} \hbar c} \rho_{g} t I_{m n: \rho \sigma}^{(2)} \mu_{\rho}^{g f} \bar{\mu}_{\sigma}^{g f} e_{3_{m}} \bar{e}_{3_{n}} \\
& =\frac{\pi I_{3}}{3 \varepsilon_{0} \hbar c} \delta_{3_{m} 3_{n}} \delta_{\rho \sigma} \mu_{\rho}^{g f} \bar{\mu}_{\sigma}^{g f} e_{3_{m}} \bar{e}_{3_{n}} \rho_{g} t \\
& =\frac{\pi I_{3}}{3 \varepsilon_{0} \hbar c}\left|\mu^{g f}\right|^{2} \rho_{g} t
\end{aligned}
$$

and so

$$
R_{2}^{1 \phi}=\frac{\pi I_{3}}{3 \varepsilon_{0} \hbar c} \rho_{g}\left|\mu^{g f}\right|^{2} .
$$


The overall rate, combining eqn (33) and (35) is thus

$$
\begin{aligned}
P(\tau) & =\int_{0}^{\tau} P_{1}^{2 \phi}(t) R_{2}^{1 \phi} \mathrm{d} t \\
& =\frac{I_{1} I_{2} I_{3} \pi \rho_{g}}{1080 \varepsilon_{0}^{3} c^{3} \hbar^{3}}\left|\mu^{\theta \delta}\right|^{2} \tau^{3}\left(\begin{array}{c}
\left(e_{1} \cdot e_{2}\right)\left(\bar{e}_{1} \cdot \bar{e}_{2}\right) \\
1 \\
\left(e_{1} \cdot \bar{e}_{2}\right)\left(e_{2} \cdot \bar{e}_{1}\right)
\end{array}\right){ }^{T}\left(\begin{array}{rrr}
10 & 0 & -2 \\
0 & 5 & 3 \\
0 & -5 & 3
\end{array}\right)\left(\begin{array}{l}
S_{\lambda \mu}^{(0)} \bar{S}_{\lambda \mu}^{(0)} \\
S_{\lambda \mu}^{(1)} \bar{S}_{\lambda \mu}^{(1)} \\
S_{\lambda \mu}^{(2)} \bar{S}_{\lambda \mu}^{(2)}
\end{array}\right)
\end{aligned}
$$

\subsubsection{Concerted Process}

Here three photons are absorbed or else two are absorbed and one is emitted, simultaneously. Using the result from eqn (9)

$$
\begin{gathered}
P_{f}(t)=\frac{2 \pi}{\hbar}\left|M_{g i}^{3 \phi}\right|^{2} \rho_{g} t \\
\left|M_{g i}^{3 \phi}\right|^{2}=\left\langle\left|\mathrm{i}\left(n_{1} n_{2} n_{3} \omega_{1} \omega_{2} \omega_{3}\right)^{1 / 2}\left(\frac{\hbar}{2 \varepsilon_{0} V}\right)^{3 / 2} T_{i j k} e_{1_{i}} e_{2_{j}} e_{3_{k}}\right|^{2}\right\rangle \\
=\frac{I_{1} I_{2} I_{3}}{8 \varepsilon_{0}^{3} c^{3}}\left\langle T_{i j k} \bar{T}_{l m n} e_{1_{i}} \bar{e}_{1_{i}} e_{2 j} \bar{e}_{2_{m}} e_{3_{k}} \bar{e}_{3_{n}}\right\rangle \\
=\frac{I_{1} I_{2} I_{3}}{8 \varepsilon_{0}^{3} c^{3}} I_{i j k l m n: \lambda \mu v o \rho \sigma}^{(6)} T_{\lambda \mu \nu} \overline{\boldsymbol{T}}_{o \rho \sigma} e_{1_{i}} \bar{e}_{1_{l}} e_{2_{j}} \bar{e}_{2_{m}} e_{3_{k}} \bar{e}_{3_{n}}
\end{gathered}
$$

where $\boldsymbol{T}_{i j k}$ is the usual three-photon absorption tensor.

Adopting similar methods to those described in section (2.3.1) with the result for $I^{(6)}$ as given by ref. (28), and irreducible tensor relations as given by ref. (29), we then obtain

$P^{3 \phi}=\frac{\pi I_{1} I_{2} I_{3}}{3360 \varepsilon_{0}^{3} c^{3} h} \rho_{\theta} t\left|\begin{array}{c}\left(e_{1} \cdot e_{2}\right)\left(e_{3} \cdot \bar{e}_{1}\right)\left(\bar{e}_{2} \cdot \bar{e}_{3}\right) \\ \left(e_{1} \cdot e_{2}\right)\left(e_{3} \cdot \bar{e}_{2}\right)\left(\bar{e}_{1} \cdot \bar{e}_{3}\right) \\ \left|e_{1} \cdot e_{2}\right|^{2} \\ \left(e_{1} \cdot e_{3}\right)\left(e_{2} \cdot \bar{e}_{1}\right)\left(\bar{e}_{2} \cdot \bar{e}_{3}\right) \\ \left|e_{1} \cdot e_{3}\right|^{2} \\ \left(e_{1} \cdot e_{3}\right)\left(e_{2} \cdot \bar{e}_{3}\right)\left(\bar{e}_{1} \cdot \bar{e}_{2}\right) \\ \left|e_{2} \cdot e_{3}\right|^{2} \\ 1 \\ \left|e_{2} \cdot \bar{e}_{3}\right|^{2} \\ \left(e_{1} \cdot \bar{e}_{2}\right)\left(e_{2} \cdot e_{3}\right)\left(\bar{e}_{1} \cdot \bar{e}_{3}\right) \\ \left|e_{1} \cdot \bar{e}_{2}\right|^{2} \\ \left(e_{1} \cdot \bar{e}_{3}\right)\left(e_{2} \cdot \bar{e}_{3}\right)\left(e_{3} \cdot \bar{e}_{1}\right) \\ \left(e_{1} \cdot \bar{e}_{3}\right)\left(e_{2} \cdot e_{3}\right)\left(\bar{e}_{1} \cdot \bar{e}_{2}\right) \\ \left(e_{1} \cdot \bar{e}_{3}\right)\left(e_{2} \cdot \bar{e}_{1}\right)\left(e_{3} \cdot \bar{e}_{2}\right) \\ \left|e_{1} \cdot \bar{e}_{3}\right|^{2}\end{array}\right|^{2}$

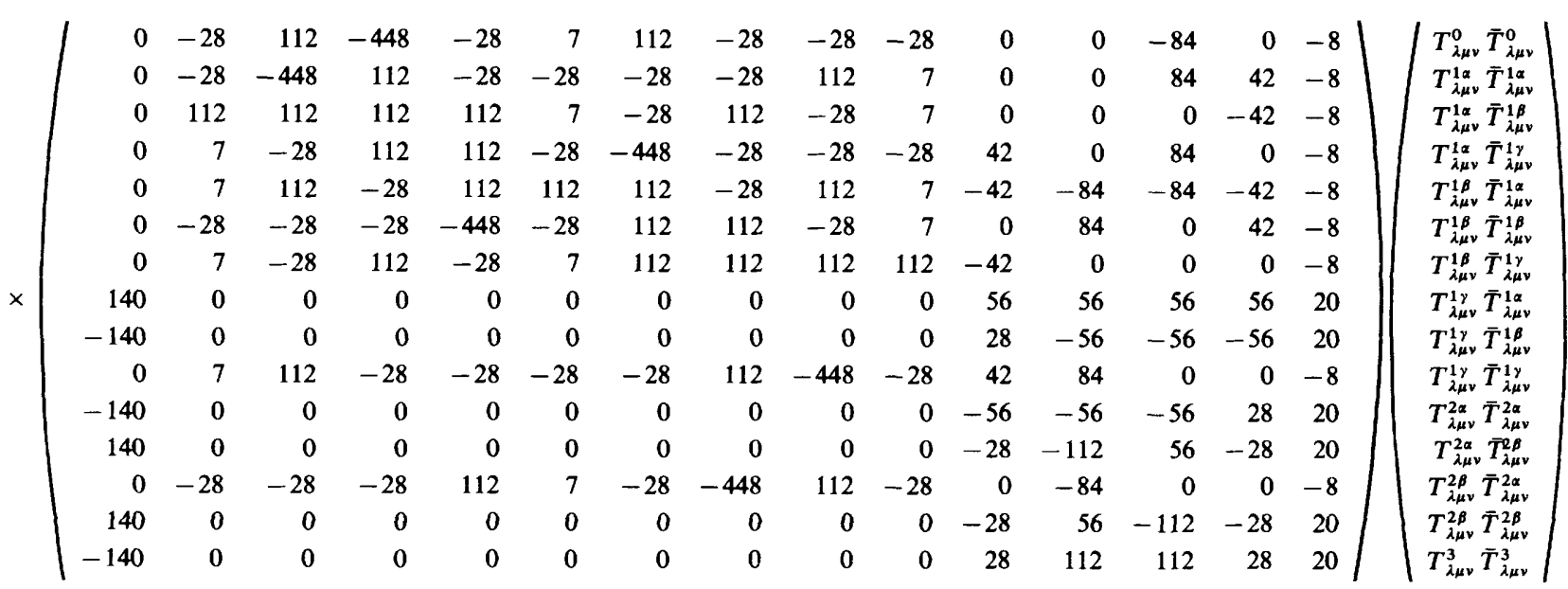




\subsubsection{Intermediate Case}

We now consider an intermediate case, where two photons are absorbed from the pump beams and then, before any rotational relaxation can occur, a probe photon is absorbed or else one photon is emitted. If there is no relaxation between the two transitions, the overall rate can be derived from the rate for the concerted three-photon process by bringing the sum of the first two photon energies into resonance with a molecular energy level, and thus essentially splitting the third-rank molecular tensor into a product of a second-rank and a first-rank tensor. This can be seen as follows.

In general the third-rank tensor $\boldsymbol{T}_{i j k}$ is given by

$$
\begin{aligned}
\boldsymbol{T}_{i j k}= & \sum_{h, f}\left(\frac{\mu_{k}^{g f} \mu_{j}^{f h} \mu_{i}^{h i}}{\left(\tilde{\varepsilon}_{f i}-\hbar \omega_{1}-\hbar \omega_{2}\right)\left(\tilde{\varepsilon}_{h i}-\hbar \omega_{1}\right)}+\frac{\mu_{j}^{g f} \mu_{k}^{f h} \mu_{i}^{h i}}{\left(\tilde{\varepsilon}_{f i}-\hbar \omega_{1}-\hbar \omega_{3}\right)\left(\tilde{\varepsilon}_{h i}-\hbar \omega_{1}\right)}\right. \\
& +\frac{\mu_{k}^{g f} \mu_{i}^{f h} \mu_{j}^{h i}}{\left(\tilde{\varepsilon}_{f i}-\hbar \omega_{1}-\hbar \omega_{2}\right)\left(\tilde{\varepsilon}_{h i}-\hbar \omega_{2}\right)}+\frac{\mu_{i}^{g f} \mu_{k}^{f h} \mu_{j}^{h i}}{\left(\tilde{\varepsilon}_{f i}-\hbar \omega_{2}-\hbar \omega_{3}\right)\left(\tilde{\varepsilon}_{h i}-\hbar \omega_{2}\right)} \\
& \left.+\frac{\mu_{j}^{g f} \mu_{i}^{f h} \mu_{k}^{h i}}{\left(\tilde{\varepsilon}_{f i}-\hbar \omega_{1}-\hbar \omega_{3}\right)\left(\tilde{\varepsilon}_{h i}-\hbar \omega_{3}\right)}+\frac{\mu_{i}^{g f} \mu_{j}^{f h} \mu_{k}^{h i}}{\left(\tilde{\varepsilon}_{f i}-\hbar \omega_{2}-\hbar \omega_{3}\right)\left(\tilde{\varepsilon}_{h i}-\hbar \omega_{3}\right)}\right)
\end{aligned}
$$

where the complex energies $\tilde{\varepsilon}_{f i}$ and $\tilde{\varepsilon}_{h i}$ incorporate damping factors and are given explicitly by $\tilde{\varepsilon}_{f i}=\varepsilon_{f i}-\frac{1}{2} \mathrm{i} \hbar \gamma_{f}$ and $\tilde{\varepsilon}_{h i}=$ $\varepsilon_{h i}-\frac{1}{2} \mathrm{i} h \gamma_{g}$. The second-rank tensor can be calculated in the same way:

$$
S_{i j}=\sum_{h}\left(\frac{\mu_{j}^{f h} \mu_{i}^{h i}}{\left(\tilde{\varepsilon}_{h i}-\hbar \omega_{1}\right)}+\frac{\mu_{i}^{f h} \mu_{j}^{h i}}{\left(\tilde{\varepsilon}_{h i}-\hbar \omega_{2}\right)}\right)
$$

Now bringing the first two photons of the three-photon process into resonance with a state $f$ gives:

$$
\varepsilon_{f i}=\hbar \omega_{1}+\hbar \omega_{2}
$$

and so the summation over $f$ in eqn (39) is dominated by one state. Because the denominator of each of the two corresponding resonance terms is very small, these two terms will be very much greater than the other terms, and hence

$$
\begin{aligned}
\boldsymbol{T}_{i j k} & \approx \sum_{h}\left(\frac{\mu_{k}^{g f} \mu_{j}^{f h} \mu_{i}^{h i}}{-\frac{1}{2} \mathrm{i} \hbar \gamma_{f}\left(\tilde{\varepsilon}_{h i}-\hbar \omega_{1}\right)}+\frac{\mu_{k}^{g f} \mu_{i}^{f h} \mu_{j}^{h i}}{-\frac{1}{2} \mathrm{i} \hbar \gamma_{f}\left(\tilde{\varepsilon}_{h i}-\hbar \omega_{2}\right)}\right) \\
& =\frac{2}{\mathrm{i} \hbar \gamma_{f}} \mu_{k}^{g f}\left[-\sum_{h}\left(\frac{\mu_{j}^{f h} \mu_{i}^{h i}}{\left(\tilde{\varepsilon}_{h i}-\hbar \omega_{1}\right)}+\frac{\mu_{i}^{f h} \mu_{j}^{h i}}{\left(\tilde{\varepsilon}_{h i}-\hbar \omega_{2}\right)}\right)\right] \\
& =K S_{i j} \mu_{k}
\end{aligned}
$$

where $K=2 / i \hbar \gamma_{f}$, and for compactness the superscript $g f$ on $\mu^{g f}$ is now dropped.

The resulting transition probability for this case will thus be the same as that for the concerted three-photon transition, but with the following substitution:

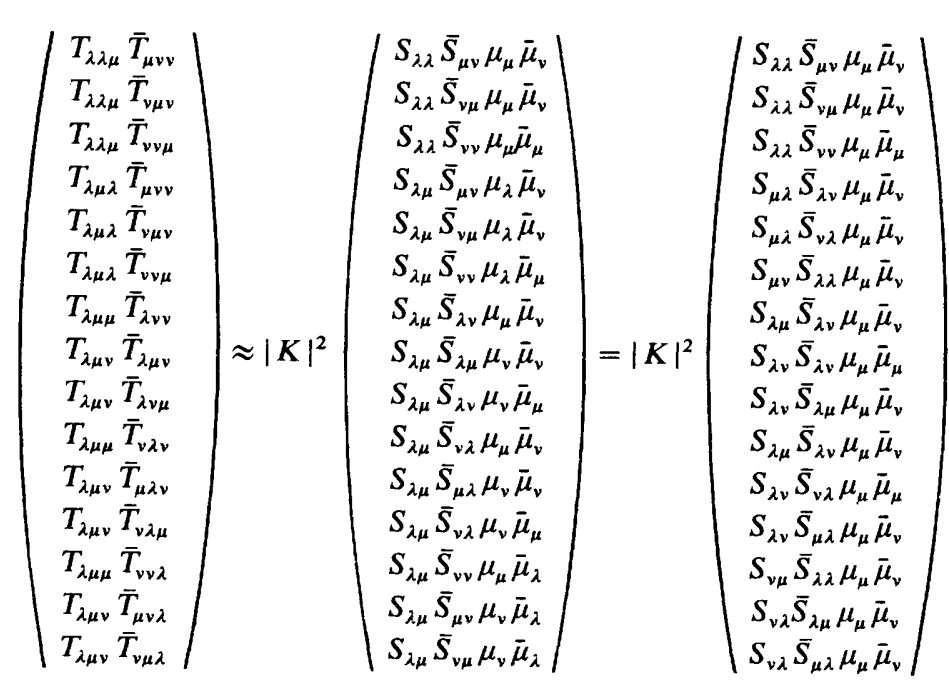


resulting in

$$
P=\frac{\pi I_{1} I_{2} I_{3}}{840 \varepsilon_{0}^{3} c^{3} h} \rho_{\theta} t\left|\begin{array}{c}
\left(e_{1} \cdot e_{2}\right)\left(e_{3} \cdot \bar{e}_{1}\right)\left(\bar{e}_{2} \cdot \bar{e}_{3}\right) \\
\left(e_{1} \cdot e_{2}\right)\left(e_{3} \cdot \bar{e}_{2}\right)\left(\bar{e}_{1} \cdot \bar{e}_{3}\right) \\
\left(e_{1} \cdot e_{2}\right)\left(\bar{e}_{1} \cdot \bar{e}_{2}\right) \\
\left(e_{1} \cdot e_{3}\right)\left(e_{2} \cdot \bar{e}_{1}\right)\left(\bar{e}_{2} \cdot \bar{e}_{3}\right) \\
\left(e_{1} \cdot e_{3}\right)\left(\bar{e}_{1} \cdot \bar{e}_{3}\right) \\
\left(e_{1} \cdot e_{3}\right)\left(e_{2} \cdot \bar{e}_{3}\right)\left(\bar{e}_{1} \cdot \bar{e}_{2}\right) \\
\left(e_{2} \cdot e_{3}\right)\left(\bar{e}_{2} \cdot \bar{e}_{3}\right) \\
1 \\
\left(e_{2} \cdot \bar{e}_{3}\right)\left(e_{3} \cdot \bar{e}_{2}\right) \\
\left(e_{1} \cdot \bar{e}_{2}\right)\left(e_{2} \cdot e_{3}\right)\left(\bar{e}_{1} \cdot \bar{e}_{3}\right) \\
\left(e_{1} \cdot e_{2}\right)\left(\bar{e}_{2} \cdot \bar{e}_{1}\right) \\
\left(e_{1} \cdot \bar{e}_{2}\right)\left(e_{2} \cdot \bar{e}_{3}\right)\left(e_{3} \cdot \bar{e}_{1}\right) \\
\left(e_{1} \cdot \bar{e}_{3}\right)\left(e_{2} \cdot e_{3}\right)\left(\bar{e}_{1} \cdot \bar{e}_{2}\right) \\
\left(e_{1} \cdot \bar{e}_{3}\right)\left(e_{2} \cdot \bar{e}_{1}\right)\left(e_{3} \cdot \bar{e}_{2}\right) \\
\left(e_{1} \cdot \bar{e}_{3}\right)\left(e_{3} \cdot \bar{e}_{1}\right)
\end{array}\right|^{2}
$$

$$
\times\left(\begin{array}{rrrrrrrrrrrrrrr}
-11 & 0 & 4 & 15 & 11 & 4 & -12 & 0 & -12 & 0 & 0 & 0 & -12 & 0 & -12 \\
-11 & 0 & 4 & 15 & 11 & 4 & -12 & 0 & -12 & 0 & 0 & 0 & -12 & 0 & -12 \\
38 & 0 & -10 & -20 & -10 & -10 & 16 & 0 & 16 & 0 & 0 & 0 & 16 & 0 & 16 \\
3 & 7 & -3 & -6 & -3 & -3 & 9 & 7 & 9 & -7 & -21 & -7 & 9 & 7 & 9 \\
3 & -7 & -3 & -6 & -3 & -3 & 9 & -7 & 9 & -7 & 21 & -7 & 9 & -7 & 9 \\
-11 & 0 & 4 & 15 & 4 & 11 & -12 & 0 & -12 & 0 & 0 & 0 & -12 & 0 & -12 \\
3 & 7 & -3 & -6 & -3 & -3 & 9 & 7 & 9 & 7 & 21 & 7 & 9 & 7 & 9 \\
-4 & 21 & 11 & 8 & 4 & 4 & -12 & 0 & -12 & 0 & -28 & 0 & -12 & 0 & -12 \\
3 & -7 & -3 & -6 & -3 & -3 & 9 & 7 & 9 & 7 & 21 & 7 & 9 & 7 & 9 \\
3 & 7 & -3 & -6 & -3 & -3 & 9 & -7 & 9 & 7 & -21 & 7 & 9 & -7 & 9 \\
-4 & -21 & 11 & 8 & 4 & 4 & -12 & 0 & -12 & 0 & 28 & 0 & -12 & 0 & -12 \\
3 & 7 & -3 & -6 & -3 & -3 & 9 & -7 & 9 & 7 & -21 & 7 & 9 & -7 & 9 \\
-11 & 0 & 4 & 15 & 4 & 11 & -12 & 0 & -12 & 0 & 0 & 0 & -12 & 0 & -12 \\
3 & 7 & -3 & -6 & -3 & -3 & 9 & 7 & 9 & -7 & -21 & -7 & 9 & 7 & 9 \\
3 & -7 & -3 & -6 & -3 & -3 & 9 & -7 & 9 & -7 & 21 & -7 & 9 & -7 & 9
\end{array}\right)\left(\begin{array}{l}
S_{\lambda \mu}^{(0)} \bar{S}_{\lambda \mu}^{(0)}|\mu|^{2} \\
S_{\lambda \mu}^{(1)} \bar{S}_{\lambda \mu}^{(1)}|\mu|^{2} \\
S_{\lambda \mu}^{(2)} S_{\lambda \mu}^{(2)}|\mu|^{2} \\
S_{\lambda \lambda}^{(0)} \bar{S}_{\mu \nu}^{(0)} \mu_{\mu} \bar{\mu}_{v} \\
S_{\lambda \lambda}^{(0)} \bar{S}_{\mu \nu}^{(2)} \mu_{\mu} \bar{\mu}_{v} \\
S_{\mu \nu}^{(2)} \bar{S}_{\lambda \lambda}^{(0)} \mu_{\mu} \bar{\mu}_{v} \\
S_{\lambda \mu}^{(0)} \bar{S}_{\lambda v}^{(0)} \mu_{\mu} \bar{\mu}_{v} \\
S_{\lambda \mu}^{(0)} \bar{S}_{\lambda \nu}^{(1)} \mu_{\mu} \bar{\mu}_{v} \\
S_{\lambda \mu}^{(0)} \bar{S}_{\lambda \nu}^{(2)} \mu_{\mu} \bar{\mu}_{v} \\
S_{\lambda \mu}^{(1)} \bar{S}_{\lambda \nu}^{(0)} \mu_{\mu} \bar{\mu}_{v} \\
S_{\lambda \mu}^{(1)} \bar{S}_{\lambda \nu}^{(1)} \mu_{\mu} \bar{\mu}_{v} \\
S_{\lambda \mu}^{(1)} \bar{S}_{\lambda \nu}^{(2)} \mu_{\mu} \bar{\mu}_{v} \\
S_{\lambda \mu}^{(2)} \bar{S}_{\lambda \nu}^{(0)} \mu_{\mu} \bar{\mu}_{v} \\
S_{\lambda \mu}^{(2)} \bar{S}_{\lambda \nu}^{(1)} \mu_{\mu} \bar{\mu}_{v} \\
S_{\lambda \mu}^{(2)} \bar{S}_{\lambda v}^{(2)} \mu_{\mu} \bar{\mu}_{v}
\end{array}\right)
$$

The molecular response tensor can be regarded as real, however, when excitation frequencies are well away from resonances other than the $\varepsilon_{f i}$ resonance. In this case;

$$
\begin{aligned}
& S_{\lambda \lambda}^{(0)} \bar{S}_{\mu \nu}^{(2)}=S_{\mu \nu}^{(2)} \bar{S}_{\lambda \lambda}^{(0)} \\
& S_{\lambda \mu}^{(0)} \bar{S}_{\lambda \nu}^{(1)}=S_{\lambda \mu}^{(1)} \bar{S}_{\lambda v}^{(0)} \text { etc. }
\end{aligned}
$$


and eqn (43) reduces to

$$
\begin{aligned}
& P=\frac{\pi I_{1} I_{2} I_{3}}{840 \varepsilon_{0}^{3} c^{3} \hbar} \rho_{g} t\left(\begin{array}{c}
\left(e_{1} \cdot e_{2}\right)\left(e_{3} \cdot \bar{e}_{1}\right)\left(\bar{e}_{2} \cdot \bar{e}_{3}\right) \\
\left(e_{1} \cdot e_{2}\right)\left(e_{3} \cdot \bar{e}_{2}\right)\left(\bar{e}_{1} \cdot \bar{e}_{3}\right) \\
\left(e_{1} \cdot e_{2}\right)\left(\bar{e}_{1} \cdot \bar{e}_{2}\right) \\
\left(e_{1} \cdot e_{3}\right)\left(e_{2} \cdot \bar{e}_{1}\right)\left(\bar{e}_{2} \cdot \bar{e}_{3}\right) \\
\left(e_{1} \cdot e_{3}\right)\left(\bar{e}_{1} \cdot \bar{e}_{3}\right) \\
\left(e_{1} \cdot e_{3}\right)\left(e_{2} \cdot \bar{e}_{3}\right)\left(\bar{e}_{1} \cdot \bar{e}_{2}\right) \\
\left(e_{2} \cdot e_{3}\right)\left(\bar{e}_{2} \cdot \bar{e}_{3}\right) \\
1 \\
\left(e_{2} \cdot \bar{e}_{3}\right)\left(e_{3} \cdot \bar{e}_{2}\right) \\
\left(e_{1} \cdot \bar{e}_{2}\right)\left(e_{2} \cdot e_{3}\right)\left(\bar{e}_{1} \cdot \bar{e}_{3}\right) \\
\left(e_{1} \cdot \bar{e}_{2}\right)\left(e_{2} \cdot \bar{e}_{1}\right) \\
\left(e_{1} \cdot \bar{e}_{2}\right)\left(e_{2} \cdot \bar{e}_{3}\right)\left(\bar{e}_{3} \cdot \bar{e}_{1}\right) \\
\left(e_{1} \cdot \bar{e}_{3}\right)\left(e_{2} \cdot e_{3}\right)\left(\bar{e}_{1} \cdot \bar{e}_{2}\right) \\
\left(e_{1} \cdot \bar{e}_{3}\right)\left(e_{2} \cdot \bar{e}_{1}\right)\left(e_{3} \cdot \bar{e}_{2}\right) \\
\left(e_{1} \cdot \bar{e}_{3}\right)\left(e_{3} \cdot \bar{e}_{1}\right)
\end{array} \bar{e}^{T}\right. \\
& \left.\left(\begin{array}{rrrrrrrrrrr}
-11 & 0 & 4 & 15 & 15 & -12 & 0 & -24 & 0 & 0 & -12 \\
-11 & 0 & 4 & 15 & 15 & -12 & 0 & -24 & 0 & 0 & -12 \\
38 & 0 & -10 & -20 & -20 & 16 & 0 & 32 & 0 & 0 & 16 \\
3 & 7 & -3 & -6 & -6 & 9 & 0 & 18 & -21 & 0 & 9 \\
3 & -7 & -3 & -6 & -6 & 9 & -14 & 18 & 21 & -14 & 9 \\
-11 & 0 & 4 & 15 & 15 & -12 & 0 & -24 & 0 & 0 & -12 \\
3 & 7 & -3 & -6 & -6 & 9 & 14 & 18 & 21 & 14 & 9 \\
-4 & 21 & 11 & 8 & 8 & -12 & 0 & -24 & -28 & 0 & -12 \\
3 & -7 & -3 & -6 & -6 & 9 & 14 & 18 & 21 & 14 & 9 \\
3 & 7 & -3 & -6 & -6 & 9 & 0 & 18 & -21 & 0 & 9 \\
-4 & -21 & 11 & 8 & 8 & -12 & 0 & -24 & 28 & 0 & -12 \\
3 & 7 & -3 & -6 & -6 & 9 & 0 & 18 & -21 & 0 & 9 \\
-11 & 0 & 4 & 15 & 15 & -12 & 0 & -24 & 0 & 0 & -12 \\
3 & 7 & -3 & -6 & -6 & 9 & 0 & 18 & -21 & 0 & 9 \\
3 & -7 & -3 & -6 & -6 & 9 & -14 & 18 & 21 & -14 & 9
\end{array}\right) \mid \begin{array}{l}
S_{\lambda \mu}^{(0)} S_{\lambda \mu}^{(0)}|\mu|^{2} \\
S_{\lambda \mu}^{(1)} S_{\lambda \mu}^{(1)}|\mu|^{2} \\
S_{\lambda \mu}^{(2)} S_{\lambda \mu}^{(2)}|\mu|^{2} \\
S_{\lambda \lambda}^{(0)} S_{\mu \nu}^{(0)} \mu_{\mu} \bar{\mu}_{v} \\
S_{\lambda \lambda}^{(0)} S_{\mu \nu}^{(2)} \mu_{\mu} \bar{\mu}_{v} \\
S_{\lambda \mu}^{(0)} S_{\lambda \nu}^{(0)} \mu_{\mu} \bar{\mu}_{\nu} \\
S_{\lambda \mu}^{(0)} S_{\lambda v}^{(1)} \mu_{\mu} \bar{\mu}_{v} \\
S_{\lambda \mu}^{(0)} S_{\lambda v}^{(2)} \mu_{\mu} \bar{\mu}_{v} \\
S_{\lambda \mu}^{(1)} S_{\lambda v}^{(1)} \mu_{\mu} \bar{\mu}_{v} \\
S_{\lambda \mu}^{(1)} S_{\lambda v}^{(2)} \mu_{\mu} \bar{\mu}_{v} \\
S_{\lambda \mu}^{(2)} S_{\lambda v}^{(2)} \mu_{\mu} \bar{\mu}_{v}
\end{array}\right)
\end{aligned}
$$

The rate equation for the intermediate case can be checked for plausibility by relating it to the two-step process. This is accomplished by rotationally averaging the $\mu$ s with respect to the molecular response tensor $S_{i j}$. Again using results derived in the Appendix and substituting this into the equation for the transition probability yields

$$
\begin{aligned}
& P(t)=\frac{\pi I_{1} I_{2} I_{3}|K|^{2}}{2520 \varepsilon_{0}^{3} c^{3} h} \rho_{g} t\left|\mu^{g f}\right|^{2}\left(\begin{array}{c}
\left(e_{1} \cdot e_{2}\right)\left(e_{3} \cdot \bar{e}_{1}\right)\left(\bar{e}_{2} \cdot \bar{e}_{3}\right) \\
\left(e_{1} \cdot e_{2}\right)\left(e_{3} \cdot \bar{e}_{2}\right)\left(\bar{e}_{1} \cdot \bar{e}_{3}\right) \\
\left|e_{1} \cdot e_{2}\right|^{2} \\
\left(e_{1} \cdot e_{3}\right)\left(e_{2} \cdot \bar{e}_{1}\right)\left(\bar{e}_{2} \cdot \bar{e}_{3}\right) \\
\left|e_{1} \cdot e_{3}\right|^{2} \\
\left(e_{1} \cdot e_{3}\right)\left(e_{2} \cdot \bar{e}_{3}\right)\left(\bar{e}_{1} \cdot \bar{e}_{2}\right) \\
\left|e_{2} \cdot e_{3}\right|^{2} \\
1 \\
\left|e_{2} \cdot \bar{e}_{3}\right|^{2} \\
\left(e_{1} \cdot \bar{e}_{2}\right)\left(e_{2} \cdot e_{3}\right)\left(\bar{e}_{1} \cdot \bar{e}_{3}\right) \\
\left|e_{1} \cdot \bar{e}_{2}\right|^{2} \\
\left(e_{1} \cdot \bar{e}_{2}\right)\left(e_{2} \cdot \bar{e}_{3}\right)\left(e_{3} \cdot \bar{e}_{1}\right) \\
\left(e_{1} \cdot \bar{e}_{3}\right)\left(e_{2} \cdot e_{3}\right)\left(\bar{e}_{1} \cdot \bar{e}_{2}\right) \\
\left(e_{1} \cdot \bar{e}_{3}\right)\left(e_{2} \cdot \bar{e}_{1}\right)\left(e_{3} \cdot \bar{e}_{2}\right) \\
\left|e_{1} \cdot \bar{e}_{3}\right|^{2}
\end{array}\right)\left(\begin{array}{rrr}
0 & 0 & 0 \\
0 & 0 & 0 \\
70 & 0 & -14 \\
0 & 0 & 0 \\
0 & 0 & 0 \\
0 & 0 & 0 \\
0 & 0 & 0 \\
0 & 35 & 21 \\
0 & 0 & 0 \\
0 & 0 & 0 \\
0 & -35 & 21 \\
0 & 0 & 0 \\
0 & 0 & 0 \\
0 & 0 & 0 \\
0 & 0 & 0
\end{array}\right)\left(\begin{array}{l}
S_{\lambda \mu}^{(0)} S_{\lambda \mu}^{(0)} \\
S_{\lambda \mu}^{(1)} S_{\lambda \mu}^{(1)} \\
S_{\lambda \mu}^{(2)} S_{\lambda \mu}^{(2)}
\end{array}\right) \\
& =\frac{\pi I_{1} I_{2} I_{3} \rho_{g}}{90 \varepsilon_{0}^{3} c^{3} \hbar^{3} \gamma_{f}^{2}} t\left|\mu^{g f}\right|^{2}\left(\begin{array}{c}
\left|e_{1} \cdot e_{2}\right|^{2} \\
1 \\
\left|e_{1} \cdot \bar{e}_{2}\right|^{2}
\end{array}\right)\left(\begin{array}{rrr}
10 & 0 & -2 \\
0 & 5 & 3 \\
0 & -5 & 3
\end{array}\right)\left(\begin{array}{c}
S_{\lambda \mu}^{(0)} S_{\lambda \mu}^{(0)} \\
S_{\lambda \mu}^{(1)} S_{\lambda \mu}^{(1)} \\
S_{\lambda \mu}^{(2)} S_{\lambda \mu}^{(2)}
\end{array}\right)
\end{aligned}
$$


but the damping factor has dimensions of (time) $)^{-1}$ and can be written;

$$
\gamma_{f}=\Gamma_{f} / t
$$

which gives

$$
P(t)=\frac{\pi I_{1} I_{2} I_{3} \rho_{g}}{90 \varepsilon_{0}^{3} c^{3} \hbar^{3} \Gamma_{f}^{2}} t^{3}\left|\mu^{g f}\right|^{2}\left(\begin{array}{c}
\left|e_{1} \cdot e_{2}\right|^{2} \\
1 \\
\left|e_{1} \cdot \bar{e}_{2}\right|^{2}
\end{array}\right)\left(\begin{array}{rrr}
10 & 0 & -2 \\
0 & 5 & 3 \\
0 & -5 & 3
\end{array}\right)\left(\begin{array}{c}
S_{\lambda \mu}^{(0)} S_{\lambda \mu}^{(0)} \\
S_{\lambda \mu}^{(1)} S_{\lambda \mu}^{(1)} \\
S_{\lambda \mu}^{(2)} S_{\lambda \mu}^{(2)}
\end{array}\right)
$$

which has exactly the same form as the rate eqn (36).

\section{Selection Rules}

For a multiphoton transition from a totally symmetric ground state, the excited-state symmetry dictates the representation under which components of the molecular tensor must transform. Therefore, for a transition to occur one or more of the irreducible tensors must transform under the irreducible representation of the final state. If the initial state is not totally symmetric then one or more of the irreducible tensors must transform under the product of the irreducible representations of the initial and final states.

To proceed further, it is necessary to determine the transformation properties of irreducible tensors under the symmetry operations of the point group appropriate for any particular molecule. The first stage involves mapping the irreducible representation of the full three-dimensional rotation-inversion group $O(3)$ onto the corresponding representations of point groups with lower symmetry. A complete listing for all the common molecular and crystallographic point groups is available elsewhere. ${ }^{30}$

Note that the molecular response tensors involve products of electric dipole transition moments, and hence the transformation properties of $\boldsymbol{S}_{i j}$ and $\boldsymbol{T}_{i j k}$ are determined by relations (47) and (48);

$$
\begin{gathered}
D^{1-} \otimes D^{1-}=D^{0+} \oplus D^{1+} \oplus D^{2+} \\
D^{1-} \otimes D^{1-} \otimes D^{1-}=D^{0-} \oplus 3 D^{1-} \oplus 2 D^{2-} \oplus D^{3-} .
\end{gathered}
$$

The weights for the two- and three-photon tensors are thus represented by $0^{+}, 1^{+}, 2^{+}$and $0^{-}, 1^{-}, 2^{-}, 3^{-}$, respectively. For each irreducible representation of any given point group there are certain combinations of tensor weights which may be non-zero. This can be illustrated by reference to the point group $T_{\mathrm{d}}$. Consider the case of concerted three-photon absorption, for which the molecular response tensor $T_{i j k}$ in general has contributions from weights $j=0,1,2$ and 3 , all with odd parity (in the electric dipole approximation). However since $\mathrm{A}_{1}$, for example, appears only in the weight-three column of odd-parity representations in table 1, it transpires that for transitions to excited states with this symmetry only weight-three contributions are in fact permitted by the selection rules. In the case of three-photon transitions to an $A_{2}$ state, by contrast, only weight-zero contributions are allowed.

The question of which tensor weights are permitted under multiphoton selection rules has a powerful influence in determining polarization behaviour. Consequently, it is helpful to classify each potential excited state in terms of the combination of allowed weights in tensors of each rank and parity. Table 2 illustrates the full results for molecules of $T_{\mathrm{d}}$ symmetry. For example from the column headed rank 3, odd parity, it is evident that in a three-beam concerted three-photon absorption process all transitions are in principle allowed, and each excited-state symmetry class is associated with a different combination of weights. This means that suitably chosen polarization conditions will, in fact, allow the complete and unambiguous symmetry classification of every line in the spectrum. By contrast, the entries under the heading of rank 2, even parity, show that two-photon transitions to excited states of symmetry $A_{2}$ are forbidden, and polarization studies cannot differentiate between the polarization behaviour of $E$ and $T_{2}$ transitions since they both carry the same weights.

Table 1. Representations of irreducible tensors in the point group $T_{\mathrm{d}}$; even-parity representations are denoted by upright characters, and odd-parity by italic characters

\begin{tabular}{|c|c|c|c|c|c|c|c|}
\hline \multirow{2}{*}{\multicolumn{2}{|c|}{ parity }} & \multicolumn{2}{|c|}{ rank 1} & \multicolumn{2}{|c|}{ rank 2} & \multicolumn{2}{|c|}{ rank 3} \\
\hline & & + & - & + & - & + & - \\
\hline \multirow[t]{5}{*}{$T_{\mathrm{d}}$} & $\mathbf{A}_{1}$ & & & (0) & & (0) & (3) \\
\hline & $A_{2}$ & & & & (0) & (3) & (0) \\
\hline & $\mathrm{E}^{2}$ & & & (2) & (2) & (2) & (2) \\
\hline & $T_{1}$ & (1) & & (1) & (2) & (13) & (23) \\
\hline & $\mathrm{T}_{2}$ & & (1) & (2) & (1) & (23) & (13) \\
\hline
\end{tabular}

\begin{tabular}{cccc}
\hline $\mathrm{D}^{(0+)}$ & $\mathrm{D}^{(1+)}$ & $\mathrm{D}^{(2+)}$ & $\mathrm{D}^{(3+)}$ \\
$D^{(0-)}$ & $D^{(1-)}$ & $D^{(2-)}$ & $D^{(3-)}$ \\
\hline $\mathrm{A}_{1}$ & $\mathrm{~T}_{1}$ & $\mathrm{E}+\mathrm{T}_{2}$ & $\mathrm{~A}_{2}+\mathrm{T}_{1}+\mathrm{T}_{2}$ \\
$A_{2}$ & $T_{2}$ & $E+T_{1}$ & $A_{1}+T_{1}+T_{2}$ \\
\hline
\end{tabular}

Table 2. Weights allowed under the irreducible representations of the point group $T_{d}$ in response tensors up to rank 3 
Table 3 provides a complete listing of the various distinguishable symmetry classes that actually arise for two- and threephoton processes in molecules belonging to the common point groups, with examples of each class. For example, in the case of two-photon absorption there are six classes (i) weights $0,1,2$, present, (ii) weights 1 and 2 alone, (iii) weights 0 and 2 alone, (iv) weight 2 alone, (v) weight 1 alone, (vi) weight 0 alone present. For three-photon processes there are eleven such classes. A suitable designation of such classes should include labels for the tensor rank and parity and it has recently been proposed ${ }^{30}$ to introduce new symmetry class labels defined as $n^{ \pm}\left(j_{i}\right)$, where $\left(j_{i}\right)$ represents the allowed weights placed in ascending order. For example, class $\mathrm{I}(\mathrm{B})$ of two-photon absorption is represented by $2^{+}(02)$, whilst the corresponding class of three-photon absorption, where only weights 0 and 2 are present, is designated $3^{-}(02)$. Previous classification schemes ${ }^{29.31-33}$ were first introduced for the experimentally simpler single-beam cases, and subsequently modified for double-beam and multiple-beam applications. Tables 4 and 5 provide the necessary correlations between the old and new classification schemes. An analysis of the allowed classes of transition for two- and three-photon absorption is given in table 6 for four of the major high-symmetry groups.

By way of example we illustrate applications of these principles for the point group $D_{6 \mathrm{~h}}$. For a totally symmetric ground state $i$, the two-photon absorption step can access the following intermediate states:

$$
f \in\left\{\mathbf{A}_{1 \mathrm{~g}}, \mathbf{A}_{2 \mathrm{~g}}, \mathrm{E}_{1 \mathrm{~g}}, \mathbf{E}_{2 \mathrm{~g}}\right\} \text {. }
$$

The interaction of the third photon with the system involves a weight $1^{-}$tensor which is spanned by $\mathrm{A}_{2 \mathrm{u}}$ and $\mathrm{E}_{1 \mathrm{u}}$ in $D_{6 \mathrm{~h}}$, and the possible final-state symmetries that can be accessed are given by:

$$
\begin{aligned}
\left(A_{2 u} \oplus E_{1 u}\right) \otimes\left(A_{1 g} \oplus A_{2 g} \oplus E_{1 g} \oplus E_{2 g}\right)= & \left(A_{2 u} \oplus E_{1 u}\right) \oplus\left(A_{1 u} \oplus E_{1 u}\right) \oplus\left(E_{1 u} \oplus A_{1 u} \oplus A_{2 u} \oplus E_{2 u}\right) \\
& \oplus\left(E_{2 u} \oplus B_{1 u} \oplus B_{2 u} \oplus E_{1 u}\right) \\
= & 2 A_{1 u} \oplus 2 A_{2 u} \oplus B_{1 u} \oplus B_{2 u} \oplus 4 E_{1 u} \oplus 2 E_{2 u} .
\end{aligned}
$$

All these final states are allowed. Table 7 shows the possible transitions: it can readily be seen that, for example, the transition $B_{2 u} \leftarrow A_{1 g}$ can involve resonance only with an intermediate state of $E_{2 g}$ symmetry.

\begin{tabular}{|c|c|c|c|}
\hline \multicolumn{2}{|c|}{ two photons } & \multicolumn{2}{|c|}{ three photons } \\
\hline class & example & class & example \\
\hline $\begin{array}{l}2^{+}(012) \\
2^{+}(12) \\
2^{+}(02) \\
2^{+}(2) \\
2^{+}(1) \\
2^{+}(0)\end{array}$ & $\begin{array}{l}\mathbf{A} / C_{1} \\
\mathbf{A}_{2} / C_{2 \mathrm{v}} \\
\mathbf{A}_{1} / C_{2 \mathrm{v}} \\
\mathbf{E}^{\prime} / D_{3 \mathrm{~h}} \\
\mathbf{A}_{2}^{\prime} / D_{3 \mathrm{~h}} \\
\mathrm{~A}_{1} / T_{\mathrm{d}}\end{array}$ & $\begin{array}{l}3^{-}(0123) \\
3^{-}(123) \\
3^{-}(023) \\
3^{-}(23) \\
3^{-}(13) \\
3^{-}(03) \\
3^{-}(02) \\
3^{-}(3) \\
3^{-}(2) \\
3^{-}(1) \\
3^{-}(0)\end{array}$ & $\begin{array}{l}\mathrm{A} / C_{1} \\
\Pi / C_{\infty} \\
\mathrm{A}_{1 \mathrm{u}} / D_{3 \mathrm{~d}} \\
\mathrm{~T}_{2 \mathrm{u}} / O_{\mathrm{h}} \\
\mathrm{T}_{1 \mathrm{u}} / O_{\mathrm{h}} \\
\mathrm{A} / T \\
\Sigma^{-} / C_{\infty \mathrm{v}} \\
\mathrm{A}_{2 \mathrm{u}} / O_{\mathrm{h}} \\
\mathrm{E}_{\mathrm{u}} / O_{\mathrm{h}} \\
\mathrm{T}_{1} / I \\
\mathrm{~A}_{1 \mathrm{u}} / O_{\mathrm{h}}\end{array}$ \\
\hline
\end{tabular}

Table 3. Symmetry classes which arise in the common molecular point groups for two- and three-photon processes, with typical examples

Table 4. Correlation of general symmetry classes for two-photon processes with an earlier classification scheme for single- and doublebeam two-photon absorption

\begin{tabular}{|c|c|c|c|c|c|c|c|}
\hline & single & double & triple & & single & double & triple \\
\hline $3^{-}(0123)$ & I & IA & $\mathbf{I A}^{\prime}$ & $3^{-}(02)$ & - & IV & IV' \\
\hline $3^{-}(123)$ & I & IA & IA & $3^{-}(3)$ & II & IIB & IIB \\
\hline $3^{-}(023)$ & II & IIA & IIA $^{\prime}$ & $3^{-}(2)$ & - & IV & IV \\
\hline $3^{-}(23)$ & II & IIA & IIA & $3^{-}(1)$ & III & III & III \\
\hline $3^{-}(13)$ & I & IB & IB & $3^{-}(0)$ & - & - & V \\
\hline $3^{-}(03)$ & II & IIB & IIB $^{\prime}$ & & & & \\
\hline
\end{tabular}

\begin{tabular}{lccccl}
\hline & single & double & & single & double \\
\hline $2^{+}(012)$ & I & IA & $2^{+}(2)$ & II & IIB \\
$2^{+}(12)$ & II & IIA & $2^{+}(1)$ & - & IV \\
$2^{+}(02)$ & I & IB & $2^{+}(0)$ & III & III \\
\hline
\end{tabular}

Table 5. Correlation of general symmetry classes for three-photon processes with earlier classification schemes appropriate for single-, doubleand triple-beam absorption 
Table 6. The allowed classes of transition for two- and three-photon absorption

\begin{tabular}{|c|c|c|c|c|c|c|c|c|c|}
\hline \multirow[b]{2}{*}{ point group } & \multicolumn{3}{|c|}{ weight } & \multirow[b]{2}{*}{ class } & \multicolumn{4}{|c|}{ weight } & \multirow[b]{2}{*}{ class } \\
\hline & $0^{+}$ & $1^{+}$ & $2^{+}$ & & $0^{-}$ & $1^{-}$ & $2^{-}$ & $3^{-}$ & \\
\hline$C_{6 \mathrm{~h}}$ & $A_{B}$ & $\begin{array}{l}A_{g} \\
E_{1 g}\end{array}$ & $\begin{array}{l}A_{g} \\
E_{1 g} \\
E_{2 g}\end{array}$ & $\begin{array}{l}2^{+}(012) \\
2^{+}(12) \\
2^{+}(2)\end{array}$ & $\mathbf{A}_{\mathbf{u}}$ & $\begin{array}{l}A_{u} \\
E_{1 u}\end{array}$ & $\begin{array}{l}A_{u} \\
E_{1 u} \\
E_{2 u}\end{array}$ & $\begin{array}{l}A_{u} \\
2 B_{u} \\
E_{1 u} \\
E_{2 u}\end{array}$ & $\begin{array}{l}3^{-}(0123) \\
3^{-}(3) \\
3^{-}(123) \\
3^{-}(23)\end{array}$ \\
\hline$D_{6 \mathrm{~h}}$ & $A_{1 g}$ & $\begin{array}{l}A_{2 g} \\
E_{1 g}\end{array}$ & $\begin{array}{l}A_{1 g} \\
E_{1 g} \\
E_{2 g}\end{array}$ & $\begin{array}{l}2^{+}(02) \\
2^{+}(1) \\
2^{+}(12) \\
2^{+}(2)\end{array}$ & $\mathbf{A}_{1 \mathrm{u}}$ & $\begin{array}{l}A_{2 u} \\
E_{1 u}\end{array}$ & $\begin{array}{l}E_{1 u} \\
E_{2 u}\end{array}$ & $\begin{array}{l}A_{2 u} \\
B_{1 u} \\
B_{2 u} \\
E_{1 u} \\
E_{2 u}\end{array}$ & $\begin{array}{l}3^{-}(02) \\
3^{-}(13) \\
3^{-}(3) \\
3^{-}(3) \\
3^{-}(123) \\
3^{-}(23)\end{array}$ \\
\hline$T_{\mathrm{h}}$ & $\mathbf{A}_{\mathrm{g}}$ & $\mathrm{T}_{\mathbf{g}}$ & $\begin{array}{l}E_{g} \\
T_{g}\end{array}$ & $\begin{array}{l}2^{+}(0) \\
2^{+}(2) \\
2^{+}(12)\end{array}$ & $\mathbf{A}_{\mathbf{u}}$ & $T_{u}$ & $\begin{array}{l}\mathrm{E}_{\mathrm{u}} \\
\mathrm{T}_{\mathrm{u}}\end{array}$ & $\begin{array}{l}A_{u} \\
2 T_{u}\end{array}$ & $\begin{array}{l}3^{-}(03) \\
3^{-}(2) \\
3^{-}(123)\end{array}$ \\
\hline$O_{\mathrm{h}}$ & $A_{1 g}$ & $T_{18}$ & $\begin{array}{l}\mathrm{E}_{\mathrm{g}} \\
\mathrm{T}_{2 \mathrm{~g}}\end{array}$ & $\begin{array}{l}2^{+}(0) \\
2^{+}(2) \\
2^{+}(2) \\
2^{+}(1)\end{array}$ & $A_{1 u}$ & $\mathrm{~T}_{1 \mathrm{u}}$ & $\begin{array}{l}\mathrm{E}_{\mathrm{u}} \\
\mathrm{T}_{2 \mathrm{u}}\end{array}$ & $\begin{array}{l}A_{2 u} \\
T_{1 u} \\
T_{2 u}\end{array}$ & $\begin{array}{l}3^{-}(0) \\
3^{-}(3) \\
3^{-}(2) \\
3^{-}(13) \\
3^{-}(23)\end{array}$ \\
\hline
\end{tabular}

Table 7. The possible final states of the system and the corresponding intermediates

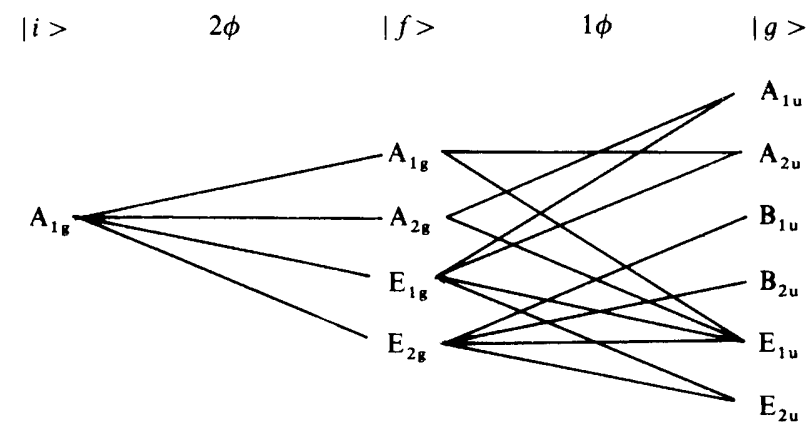

\section{Polarization Ratios}

In many chemical systems the photoselection process will be neither two-photon absorption followed by an uncorrelated single-photon transition, nor a concerted three-photon interaction, but a process with characteristics somewhere between these two extremes. A parameter is therefore required to indicate where in this dynamical spectrum the process lies. The measurement of polarization ratios can be used for this purpose. Again this can be illustrated by reference to the example from the previous section, of a $B_{2 u} \leftarrow A_{1 g}$ transition in a $D_{6 h}$ molecule.

We first consider the sequential case, where the only possible symmetry for the two-photon resonance state is $\mathrm{E}_{2 \mathrm{~g}}$. From table 6 , the only tensor weight involved in the two-photon step is $2^{+}$, and so the transition probability is given by:

$$
P(t)=\frac{I_{1} I_{2} I_{3} \pi \rho_{g}}{1080 \varepsilon_{0}^{3} c^{3} \hbar^{3}}\left|\mu^{g f}\right|^{2}\left(\begin{array}{c}
\left(e_{1} \cdot e_{2}\right)\left(\bar{e}_{1} \cdot \bar{e}_{2}\right) \\
1 \\
\left(e_{1} \cdot \bar{e}_{2}\right)\left(e_{2} \cdot \bar{e}_{1}\right)
\end{array}\right)\left(\begin{array}{rrr}
10 & 0 & -2 \\
0 & 5 & 3 \\
0 & -5 & 3
\end{array}\right)\left(\begin{array}{c}
0 \\
0 \\
S_{\lambda \mu}^{(2)} \bar{S}_{\lambda \mu}^{(2)}
\end{array}\right) t^{3} .
$$

If photons one and two are derived from a single laser beam and are plane-polarized in the same direction then we obtain;

$$
P(t)=\frac{I_{1} I_{2} I_{3} \pi \rho_{g}}{270 \varepsilon_{0}^{3} c^{3} \hbar^{3}}\left|\mu^{g f}\right|^{2} t^{3}\left(S_{\lambda \mu}^{(2)} \bar{S}_{\lambda \mu}^{(2)}\right)
$$

Because this probability does not depend on the polarization vector of the third photon, the ratio of the transition probability for the case where this photon is polarized parallel to the first laser beam, relative to the case where it is polarized perpendicular, is 1 . 
We now consider the same $B_{2 u} \leftarrow A_{1 g}$ transition as a concerted three-photon process. Only tensors of weight $3^{-}$are involved in this transition. Considering the first two photons, again plane-polarized in the same direction, gives the transition probability as:

$$
P(t)=\frac{I_{1} I_{2} I_{3} \pi \rho_{g}}{3360 \varepsilon_{0}^{3} c^{3} h} t\left(\begin{array}{c}
\left(e_{3} \cdot \bar{e}_{1}\right)\left(\bar{e}_{1} \cdot \bar{e}_{3}\right) \\
\left(e_{3} \cdot \bar{e}_{1}\right)\left(\bar{e}_{1} \cdot \bar{e}_{3}\right) \\
1 \\
\left(e_{1} \cdot e_{3}\right)\left(e_{1} \cdot \bar{e}_{3}\right) \\
\left(e_{1} \cdot e_{3}\right)\left(\bar{e}_{1} \cdot \bar{e}_{3}\right) \\
\left(e_{1} \cdot e_{3}\right)\left(e_{1} \cdot \bar{e}_{3}\right) \\
\left(e_{1} \cdot e_{3}\right)\left(\bar{e}_{1} \cdot \bar{e}_{3}\right) \\
1 \\
\left(e_{1} \cdot \bar{e}_{3}\right)\left(e_{3} \cdot \bar{e}_{1}\right) \\
\left(e_{1} \cdot e_{3}\right)\left(\bar{e}_{1} \cdot \bar{e}_{3}\right) \\
1 \\
\left(e_{1} \cdot \bar{e}_{3}\right)\left(e_{3} \cdot \bar{e}_{1}\right) \\
\left(e_{1} \cdot \bar{e}_{3}\right)\left(e_{1} \cdot e_{3}\right) \\
\left(e_{1} \cdot \bar{e}_{3}\right)\left(e_{3} \cdot \bar{e}_{1}\right) \\
\left(e_{1} \cdot \bar{e}_{3}\right)\left(e_{3} \cdot \bar{e}_{1}\right)
\end{array}\right)\left(\begin{array}{c}
-8 \\
-8 \\
-8 \\
-8 \\
-8 \\
-8 \\
-8 \\
20 \\
20 \\
-8 \\
20 \\
20 \\
-8 \\
20 \\
20
\end{array}\right)\left(T_{\lambda \mu v}^{(3)} \bar{T}_{\lambda \mu v}^{(3)}\right)
$$

If the third photon is polarized parallel to the first two, i.e. $e_{1} \equiv e_{3}$ and $e_{1} \cdot e_{3}=1$, we obtain

$$
\begin{aligned}
& P_{1}(t)=\frac{I_{1} I_{2} I_{3} \pi \rho_{g}}{3360 \varepsilon_{0}^{3} c^{3} \hbar} t\left(\begin{array}{c}
1 \\
1 \\
1 \\
1 \\
1 \\
1 \\
1 \\
1 \\
1 \\
1 \\
1 \\
1 \\
1 \\
1 \\
1 \\
1 \\
1
\end{array}\right)^{T}\left(\begin{array}{r}
-8 \\
-8 \\
-8 \\
20 \\
-8 \\
20 \\
20 \\
-8 \\
20 \\
20
\end{array}\right)\left(T_{\lambda \mu \nu}^{(3)} \bar{T}_{\lambda \mu \nu}^{(3)}\right) \\
& =\frac{I_{1} I_{2} I_{3} \pi \rho_{g}}{70 \varepsilon_{0}^{3} c^{3} \hbar} t\left(T_{\lambda \mu \nu}^{(3)} \bar{T}_{\lambda \mu \nu}^{(3)}\right) .
\end{aligned}
$$

However, with the third photon polarized perpendicular to the first two, i.e. $e_{1} \cdot e_{3}=0$, we have

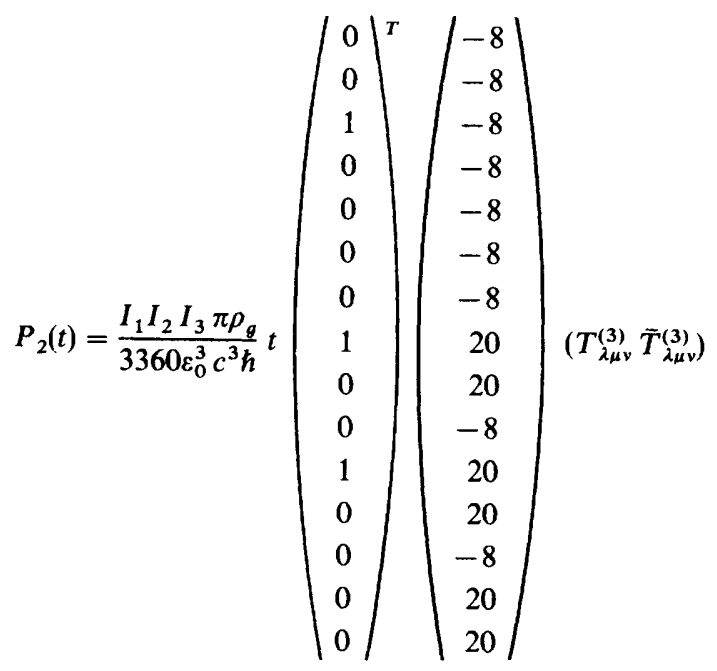

$$
\begin{aligned}
& =\frac{I_{1} I_{2} I_{3} \pi \rho_{g}}{105 \varepsilon_{0}^{3} c^{3} h} t\left(T_{\lambda \mu v}^{(3)} \bar{T}_{\lambda \mu \nu}^{(3)}\right)
\end{aligned}
$$


The above polarization ratio results apply just as equally to emission of the third photon as to absorption. The former type of concerted three-photon interaction represents a non-linear scattering process, the term hyper-Raman scattering being applied to the specific case where the two annihilated photons have the same frequency. The $3 / 2$ polarization ratio for the case of a $3^{-}(3)$ transition can, in fact, be identified from eqn (5.13) and (5.14) of ref. (34). In the experimental measurement of the polarization of fluorescence following two-photon excitation by a plane-polarized laser beam, it therefore follows that for transitions of symmetry class $3^{-}(3)$ the ratio of the intensity parallel to the exciting beam relative to that perpendicular to it should lie between 1.0 and 1.5. The location of the ratio within this range thereby provides information on the extent of rotational motion, if any, intervening between the absorption and emission stages of the process. Where the process is a two-photon resonant interaction and the intermediate stage $|f\rangle$ is physically realized, the polarization ratio has a value of 1.0; where there is no resonance and the process is one of non-linear scattering, a value of 1.5 is obtained.

Similar arguments can usefully be applied to transitions of other symmetry classes, and further information is afforded by two-beam studies of the signal dependence on the angle between the polarizations of the absorbed photons. ${ }^{29}$

\section{Appendix}

The second-rank tensor products of eqn (42) can be expressed in terms of irreducible tensors. To assist in determining the relevant irreducible tensors, a shorthand notation can be introduced. This involves representing each second-rank tensor $\boldsymbol{S}$ by a box, with dashes and crosses denoting index parameters; - means repeated indices (hence implied summation) and $\mathbf{x x}$ means hanging ones. Thus, $\mathrm{x}+\mathrm{x}$ represents say $S_{\lambda \mu} S_{\lambda v}$ with the repeated $\lambda$ contracted between the two tensors.

All the possible arrangements for the indices on the tensor products are, therefore,

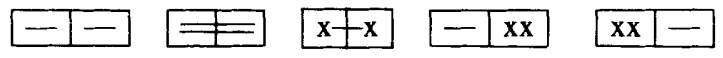

and their constituent irreducible parts can be derived as follows.

$-1-$ :

Only one irreducible tensor product is needed for this case, because;

$$
S_{\lambda \lambda} \bar{S}_{\mu \mu}=3 S_{\lambda \mu}^{(0)} \bar{S}_{\lambda \mu}^{(0)}
$$

All cross products between different weights of the irreducible tensors will be zero so that there are only three products that may be finite-valued:

$$
\mathrm{x}+\mathrm{x} \text { : } \quad S_{\lambda \mu}^{(0)} \bar{S}_{\lambda \mu}^{(0)}, S_{\lambda \mu}^{(1)} \bar{S}_{\lambda \mu}^{(1)} \text { and } S_{\lambda \mu}^{(2)} \bar{S}_{\lambda \mu}^{(2)} \text {. }
$$

Here all the possible combinations of weights may be finite-valued and so nine irreducible tensor products will be needed, all of the form

with $a, b \in\{0,1,2\}$.

$$
S_{\lambda \mu}^{(a)} \bar{S}_{\lambda v}^{(b)}
$$

\begin{tabular}{l|l|}
\hline & $\mathbf{x x}$ \\
\hline
\end{tabular}

The weight 1 and 2 components of the first tensor will be zero, because of the repeated index. The weight 0 and 1 combination will vanish on contraction with $\mu_{\mu}^{g f} \bar{\mu}_{v}^{g f}$ since with real $\mu^{g f}$ the latter is symmetric whereas $\bar{S}_{\mu \nu}^{(1)}$ is antisymmetric with respect to the indices $\mu, v$. Hence only two more tensor products arise:

\begin{tabular}{|l|l:}
\hline $\mathbf{x x}$ & - : \\
\hline
\end{tabular}

$$
S_{\lambda \lambda}^{(0)} \bar{S}_{\mu \nu}^{(0)} \text { and } S_{\lambda \lambda}^{(0)} \bar{S}_{\mu \nu}^{(2)}
$$

This case is analogous to $-\quad \mathbf{x x}$, but

so that only one more product is required:

$$
S_{\mu \nu}^{(0)} \bar{S}_{\lambda \lambda}^{(0)}=S_{\lambda \lambda}^{(0)} \bar{S}_{\mu \nu}^{(0)}
$$

$$
S_{\mu v}^{(2)} \bar{S}_{\lambda \lambda}^{(0)}
$$

Using this method facilitates the derivation of the following equation relating the molecular response parameters of equation

(42) to their irreducible counterparts:

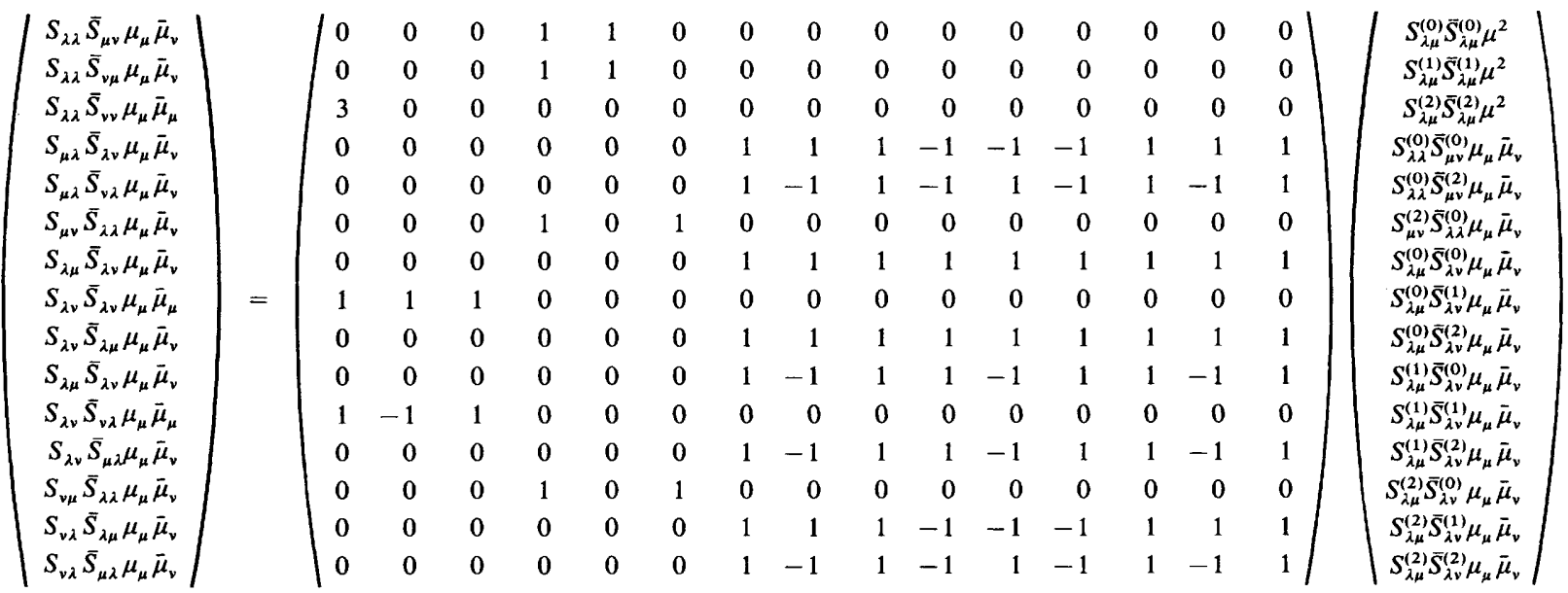


This result is similar to the one for the concerted three-photon process, eqn (38), where 15 irreducible tensor products also arise. When the tensors $S$ and $\boldsymbol{\mu}$ are real this equation reduces to one depending on 11 tensor products;

$$
\left(\begin{array}{l}
S_{\lambda \lambda} \bar{S}_{\mu \nu} \mu_{\mu} \bar{\mu}_{v} \\
S_{\lambda \lambda} \bar{S}_{v \mu} \mu_{\mu} \bar{\mu}_{v} \\
S_{\lambda \lambda} \bar{S}_{v v} \mu_{\mu} \bar{\mu}_{\mu} \\
S_{\mu \lambda} \bar{S}_{\lambda v} \mu_{\mu} \bar{\mu}_{v} \\
S_{\mu \lambda} \bar{S}_{v \lambda} \mu_{\mu} \bar{\mu}_{v} \\
S_{\mu \nu} \bar{S}_{\lambda \lambda} \mu_{\mu} \bar{\mu}_{v} \\
S_{\lambda \mu} \bar{S}_{\lambda v} \mu_{\mu} \bar{\mu}_{v} \\
S_{\lambda \nu} \bar{S}_{\lambda \nu} \mu_{\mu} \bar{\mu}_{\mu} \\
S_{\lambda \nu} \bar{S}_{\lambda \mu} \mu_{\mu} \bar{\mu}_{v} \\
S_{\lambda \mu} \bar{S}_{\lambda \nu} \mu_{\mu} \bar{\mu}_{v} \\
S_{\lambda v} \bar{S}_{v \lambda} \mu_{\mu} \bar{\mu}_{\mu} \\
S_{\lambda \nu} \bar{S}_{\mu \lambda} \mu_{\mu} \bar{\mu}_{v} \\
S_{v \mu} \bar{S}_{\lambda \lambda} \mu_{\mu} \bar{\mu}_{v} \\
S_{v \lambda} \bar{S}_{\lambda \mu} \mu_{\mu} \bar{\mu}_{v} \\
S_{v \lambda} \bar{S}_{\mu \lambda} \mu_{\mu} \bar{\mu}_{v}
\end{array}\right)=\left(\begin{array}{rrrrrrrrrrr}
0 & 0 & 0 & 1 & 1 & 0 & 0 & 0 & 0 & 0 & 0 \\
0 & 0 & 0 & 1 & 1 & 0 & 0 & 0 & 0 & 0 & 0 \\
3 & 0 & 0 & 0 & 0 & 0 & 0 & 0 & 0 & 0 & 0 \\
0 & 0 & 0 & 0 & 0 & 1 & 0 & 2 & -1 & 0 & 1 \\
0 & 0 & 0 & 0 & 0 & 1 & -2 & 2 & 1 & -2 & 1 \\
0 & 0 & 0 & 1 & 1 & 0 & 0 & 0 & 0 & 0 & 0 \\
0 & 0 & 0 & 0 & 0 & 1 & 2 & 2 & 1 & 2 & 1 \\
1 & 1 & 1 & 0 & 0 & 0 & 0 & 0 & 0 & 0 & 0 \\
0 & 0 & 0 & 0 & 0 & 1 & 2 & 2 & 1 & 2 & 1 \\
0 & 0 & 0 & 0 & 0 & 1 & 0 & 2 & -1 & 0 & 1 \\
1 & -1 & 1 & 0 & 0 & 0 & 0 & 0 & 0 & 0 & 0 \\
0 & 0 & 0 & 0 & 0 & 1 & 0 & 2 & -1 & 0 & 1 \\
0 & 0 & 0 & 1 & 1 & 0 & 0 & 0 & 0 & 0 & 0 \\
0 & 0 & 0 & 0 & 0 & 1 & 0 & 2 & -1 & 0 & 1 \\
0 & 0 & 0 & 0 & 0 & 1 & -2 & 2 & 1 & -2 & 1
\end{array}\right)\left(\begin{array}{l}
S_{\lambda \mu}^{(0)} S_{\lambda \mu}^{(0)} \mu^{2} \\
S_{\lambda \mu}^{(1)} S_{\lambda \mu}^{(1)} \mu^{2} \\
S_{\lambda \mu}^{(2)} S_{\lambda \mu}^{(2)} \mu^{2} \\
S_{\lambda \lambda}^{(0)} S_{\mu \nu}^{(0)} \mu_{\mu} \mu_{v} \\
S_{\lambda \lambda}^{(0)} S_{\mu \nu}^{(2)} \mu_{\mu} \mu_{v} \\
S_{\lambda \mu}^{(0)} S_{\lambda \nu}^{(0)} \mu_{\mu} \mu_{v} \\
S_{\lambda \mu}^{(0)} S_{\lambda \lambda}^{(1)} \mu_{\mu} \mu_{v} \\
S_{\lambda \mu}^{(0)} S_{\lambda \nu}^{(2)} \mu_{\mu} \mu_{v} \\
S_{\lambda \mu}^{(1)} S_{\lambda \nu}^{(1)} \mu_{\mu} \mu_{v} \\
S_{\lambda \mu}^{(1)} S_{\lambda \nu}^{(2)} \mu_{\mu} \mu_{v} \\
S_{\lambda \mu}^{(2)} S_{\lambda \nu}^{(2)} \mu_{\mu} \mu_{v}
\end{array}\right)
$$

Rotationally averaging the $\mu \mathrm{s}$ with respect to the molecular response tensor $\boldsymbol{S}$, i.e. allowing complete rotational relaxation to occur between the two- and one-photon steps, enables us to compare the rate for three-photon photoselection with that for a two-step process. The required result is as follows:

$$
\left(\begin{array}{l}
S_{\lambda \lambda} \bar{S}_{\mu v} \mu_{\mu} \bar{\mu}_{v} \\
S_{\lambda \lambda} \bar{S}_{v \mu} \mu_{\mu} \bar{\mu}_{v} \\
S_{\lambda \lambda} \bar{S}_{v v} \mu_{\mu} \bar{\mu}_{\mu} \\
S_{\mu \lambda} \bar{S}_{\lambda v} \mu_{\mu} \bar{\mu}_{v} \\
S_{\mu \lambda} \bar{S}_{v \lambda} \mu_{\mu} \bar{\mu}_{v} \\
S_{\mu v} \bar{S}_{\lambda \lambda} \mu_{\mu} \bar{\mu}_{v} \\
S_{\lambda \mu} \bar{S}_{\lambda v} \mu_{\mu} \bar{\mu}_{v} \\
S_{\lambda v} \bar{S}_{\lambda v} \mu_{\mu} \bar{\mu}_{\mu} \\
S_{\lambda v} \bar{S}_{\lambda \mu} \mu_{\mu} \bar{\mu}_{v} \\
S_{\lambda \mu} \bar{S}_{\lambda v} \mu_{\mu} \bar{\mu}_{v} \\
S_{\lambda v} \bar{S}_{v \lambda} \mu_{\mu} \bar{\mu}_{\mu} \\
S_{\lambda v} \bar{S}_{\mu \lambda} \mu_{\mu} \bar{\mu}_{v} \\
S_{v \mu} \bar{S}_{\lambda \lambda} \mu_{\mu} \bar{\mu}_{v} \\
S_{v \lambda} \bar{S}_{\lambda \mu} \mu_{\mu} \bar{\mu}_{v} \\
S_{v \lambda} \bar{S}_{\mu \lambda} \mu_{\mu} \bar{\mu}_{v}
\end{array}\right)=\left(\begin{array}{rrr}
3 & 0 & 0 \\
3 & 0 & 0 \\
9 & 0 & 0 \\
1 & -1 & 1 \\
1 & 1 & 1 \\
3 & 0 & 0 \\
1 & 1 & 1 \\
3 & 3 & 3 \\
1 & 1 & 1 \\
1 & -1 & 1 \\
3 & -3 & 3 \\
1 & -1 & 1 \\
3 & 0 & 0 \\
1 & -1 & 1 \\
1 & 1 & 1
\end{array}\right)\left(\begin{array}{l}
S_{\lambda \mu}^{(0)} \bar{S}_{\lambda \mu}^{(0)} \\
S_{\lambda \mu}^{(1)} \bar{S}_{\lambda \mu}^{(1)} \\
S_{\lambda \mu}^{(2)} \bar{S}_{\lambda \mu}^{(2)}
\end{array}\right)\left|\mu^{g f}\right|^{2}
$$

\section{References}

A. C. Albrecht, J. Mol. Spectrosc., 1961, 6, 84.

2 P. P. Feofilov, The Physical Basis of Polarized Emission (CB, New York, 1961).

3 J. S. Baskin, P. M. Felker and A. H. Zewail, J. Chem. Phys., 1986, 84, 4708.

4 G. S. Beddard, T. Doust and J. Hudales, Nature (London), 1981, 294, 145.

5 G. S. Beddard, Biochem. Soc. Trans., 1986, 14, 827.

6 T. J. Chuang and K. B. Eisenthal, Chem. Phys. Lett., 1971, 11, 368.

7 T. Tao, Biopolymers, 1969, 8, 609.

8 C. V. Shank and E. P. Ippen, Appl. Phys. Lett., 1975, 26, 62.

9 Z. Von Jena and H. E. Lessing, Chem. Phys. Lett., 1981, 78, 187.

10 M. W. Dowley, K. B. Eisenthal and W. L. Peticolas, J. Phys. Chem., 1967, 47, 1609.

11 W. M. McClain, J. Chem. Phys., 1972, 57, 2264.

12 W. M. McClain, J. Chem. Phys., 1973, 58, 324.

13 D. Magde, J. Chem. Phys., 1978, 68, 3717.

14 J. R. Cable and A. C. Albrecht, J. Chem. Phys., 1986, 85, 3155.

15 T. W. Scott, K. S. Haber and A. C. Albrecht, J. Chem. Phys., 1983, 78, 150.

16 J. R. Cable and A. C. Albrecht, J. Chem. Phys., 1986, 85, 3145.

17 N. Melikechi and L. Allen, J. Phys. B, 1987, 20, 6207.

18 A. C. Kummel, G. O. Sitz and R. N. Zare, J. Chem. Phys., 1988, 88, 6707

19 A. C. Kummel, G. O. Sitz and R. N. Zare, J. Chem. Phys., 1988, 88, 7357.

20 M. P. Docker, Chem. Phys., 1988, 125, 185.

21 G. R. Fleming, Chemical Applications of Ultrafast Spectroscopy (Pergamon Press, Oxford, 1986)

22 R. N. Dixon, J. M. Bayley and M. N. R. Ashfold, Chem. Phys., 1984, 84, 21.

23 T. R. Dyke, G. R. Tomasevich, W. Klempere and W. Falconer, J. Chem. Phys., 1972, 57, 2277.

24 D. L. Andrews, J. Phys. B, 1978, 11, 2655. 
25 D. L. Andrews, J. Phys. B, 1977, 10, L659.

26 W. A. McClean and S. Swain, J. Phys. B, 1978, 11, L515.

27 W. A. McClean and S. Swain, J. Phys. B, 1979, 12, 2291.

28 D. L. Andrews and T. Thirunamachandran, J. Chem. Phys., 1977, 67, 5026

29 D. L. Andrews, and P. J. Wilkes, J. Chem. Phys., 1985, 83. 2009.

30 D. L. Andrews, Spectrochim. Acta, Part A, 1990, 46, 871

31 D. L. Andrews and W. A. Ghoul, J. Chem. Phys., 1981, 75, 530.

32 D. L. Andrews, J. Chem. Phys., 1982, 77, 2831.

33 D. L. Andrews, J. Chem. Phys., 1983, 78, 1731.

34 D. L. Andrews and T. Thirunamachandran, J. Chem. Phys., 1978, 68, 2941.

Paper 0/01127D; Received 14th March, 1990 